\title{
The magnitude and effects of extreme solar particle events
}

\author{
Piers Jiggens*, Marc-Andre Chavy-Macdonald, Giovanni Santin, Alessandra Menicucci, Hugh Evans, and Alain Hilgers \\ European Space Research and Technology Centre (ESTEC), Space Environment and Effects Section Keperlaan 1, \\ 2200AG Noordwijk, The Netherlands \\ *Corresponding author: e-mail: piers.jiggens@esa.int
}

Received 18 September 2013 / Accepted 28 April 2014

\begin{abstract}
The solar energetic particle (SEP) radiation environment is an important consideration for spacecraft design, spacecraft mission planning and human spaceflight. Herein is presented an investigation into the likely severity of effects of a very large Solar Particle Event (SPE) on technology and humans in space. Fluences for SPEs derived using statistical models are compared to historical SPEs to verify their appropriateness for use in the analysis which follows. By combining environment tools with tools to model effects behind varying layers of spacecraft shielding it is possible to predict what impact a large SPE would be likely to have on a spacecraft in Near-Earth interplanetary space or geostationary Earth orbit. Also presented is a comparison of results generated using the traditional method of inputting the environment spectra, determined using a statistical model, into effects tools and a new method developed as part of the ESA SEPEM Project allowing for the creation of an effect time series on which statistics, previously applied to the flux data, can be run directly. The SPE environment spectra is determined and presented as energy integrated proton fluence $\left(\mathrm{cm}^{-2}\right)$ as a function of particle energy (in MeV). This is input into the SHIELDOSE-2, MULASSIS, NIEL, GRAS and SEU effects tools to provide the output results. In the case of the new method for analysis, the flux time series is fed directly into the MULASSIS and GEMAT tools integrated into the SEPEM system. The output effect quantities include total ionising dose (in rads), non-ionising energy loss ( $\mathrm{MeV} \mathrm{g}^{-1}$ ), single event upsets (upsets/bit) and the dose in humans compared to established limits for stochastic (or cancer-causing) effects and tissue reactions (such as acute radiation sickness) in humans given in grey-equivalent and sieverts respectively.
\end{abstract}

Key words. SEP - radiation - human spaceflight - Carrington event - extreme events

\section{Introduction}

In the design process for spacecraft and missions the space radiation environment is an important factor. Components must be sufficiently protected, either by design or shielding, to withstand the harshness of this environment. There are three sources of particle radiation to consider for the majority of space missions:

1. The Earth's trapped radiation belts which are timedependent with driving inputs depending on the conditions in near-Earth interplanetary space;

2. The galactic cosmic ray (GCR) background which slowly varies over the solar cycle anti-correlated with solar activity as the increased influence of the heliospheric magnetic field results in greater particle attenuation at solar maximum (the impact is greater for low energy radiation);

3. The solar energetic particle (SEP) population which is sporadic depending on solar events such as shocks driven by fast and wide coronal mass ejections (CMEs) with a greater frequency of solar particle events (SPEs) seen during solar maximum as opposed to solar minimum.

In this study the focus is placed on the last of these sources which is dominant over short timescales (in the $\mathrm{MeV}$ to $\mathrm{GeV}$ energy range) when extreme SPEs occur for all but low altitude, low latitude Earth-orbiting spacecraft. The case study applied is for a 9-month manned mission to EML-2 (the Earth-Moon Lagrange point) in near-Earth interplanetary space but the environments derived based on confidence levels are also extrapolated to their likely rate of occurrence.

There are different guidelines for how to determine a worstcase SPE spectrum. In work by King (1974) the SPE of August 1972 was found to be anomalously large in comparison with the other events of solar cycle 20 dominating the cumulative SEP contribution for that cycle. This is probably the largest SPE of the space age for energies relevant to spacecraft components. An earlier event in February 1956 observed by neutron monitors (see Dorman et al. 2004) is thought to have had an extremely hard spectrum making it more significant for effects resulting from incident particles of higher energies $(>500 \mathrm{MeV})$. These two SPEs formed the basis for the worstcase events produced for the CREME-86 model (Adams 1986). However, it is difficult to derive (in the case of February 1956) or validate (in the case of August 1972) the fluxes for these SPEs. The typical worst-case event used in CREME-96 (Tylka et al. 1997) is that of October 1989 which is the largest SPE of the past 40 years in the $5 \mathrm{MeV}-100 \mathrm{MeV}$ energy range. These examples highlight a problem; that an SPE which provides the worst-case short-term environment for one spacecraft component may not provide the worst case short-term environment for another component with different characteristics and shielding geometry.

Xapsos et al. (1999) defined a "worst-case" SPE to be applied for any given mission depending on the mission duration and a user-defined confidence level based on Poisson statistics and a truncated power law fit to the event fluences. The worst-case fluences are energy-dependent and the methodology 
has been reproduced for software such as SPENVIS. The confidence level specifies the probability required that this event fluence shall not be exceeded meaning that it is not the absolute worst-case but a value with a (usually) small risk of being exceeded. However, the distribution used by Xapsos et al. also predicts an absolute worst-case labelled as the "design limit" which the authors state there is zero-risk of being exceeded for a specified energy. The existence and value of such a limit is highly contentious due to the lack of data for very large fluence SPEs; other distributions applied to SPE fluences do not define such a limit. A method for calculating a "worst-case" event fluence for a given mission length and confidence level was also reported by Jiggens et al. (2012) following the Virtual Timelines methodology combining a Lévy distribution fit to the event waiting times (see Jiggens \& Gabriel 2009) and a power law with exponential cut-off fit to the SPE fluences first applied by Nymmik (2007). Here, no absolute worst-case is predicted. In terms of probabilistic models the meaning of "worst-case" is usually "a value which one can be $\mathrm{X} \%$ certain will not be exceeded by any single event over the mission" but for the sake of brevity the term "X\% worst-case" is often used. Note that such methods can be applied to SPE integrated flux (fluence) and peak flux.

Although any output spectrum calculated using probabilistic models is, in reality, a combination of possible worst-case events, these methods provide a more coherent statistical approach than taking a single historical example event as the worst-case. In Section 2 a comparison is made between SPE spectra derived from statistical modelling and observed historical events. The statistical events' spectra are later used for the determination of effects.

In the standard methodology once a spectrum has been determined it can then be used as input into an effects tool depending on the effect in which the spacecraft or mission designer is interested. In this work the following outputs are derived:

1. Total Ionising Dose (TID) which is an important parameter for electronic component and material degradation over the duration of a mission;

2. Non-Ionising Energy Loss (NIEL) which is a parameter to describe damage to target lattice structures resulting in material degradation or damage to optics;

3. Single Event Upsets (SEUs) resulting in lost data, required instrument hard reset or in extreme cases the loss of an instrument;

4. Stochastic effects in humans which can significantly increase the likelihood of an astronaut developing late pathologies (e.g., cancer);

5. Tissue reactions (formerly referred to as as deterministic effects) in humans which can cause short-term effects such as cataracts and acute radiation sickness.

Also introduced is a new method (available on the SEPEM system) for deriving these quantities by first fixing the shielding geometry and then using the effects tools to produce a time series - not of incident particle flux but rather of TID, NIEL, etc. - before applying the statistical tools. This allows focus to be placed on the time periods important for the specific effect for humans or specific components and eliminates the need for multiple statistical model runs for different particle energies and the possible exaggeration of the worst-case through a combination of these outputs.

\section{Large SPEs}

During the space age there have been several large SPEs greatly enhancing the particle radiation environment by orders of magnitude thereby resulting in particle radiation-related effects. The causes of many of these SPEs have been studied in the literature with CMEs and associated shocks being identified as the main driver for these enhancements under the current paradigm (see Kahler 2003). The CME shock characteristics which differentiate between major and minor SPEs are described variously in the literature as the width, speed (Kahler 2001), solar origin relative to the Earth (Lario et al. 2006) and interaction with other CMEs (Gopalswamy et al. 2004) and shall not be discussed further in this work. The focus herein is in the resulting SPE spectra and the possibility of more severe events than have been measured to date. First, statistical model outputs are compared to the recorded event spectra in order to justify the use of higher confidence level outputs from these models as examples of possible "extreme" SPEs.

\subsection{August 1972}

The SPE of August 1972 is often cited as a prototypical worstcase SPE which could result in severe health risks to astronauts on EVA or even death. It occurred between the NASA Apollo 16 and Apollo 17 lunar missions. Figure 1 shows the flux-time profiles taken from the IMP-5 spacecraft in four integral energy channels. Some processing has been performed to remove spikes and interpolate data gaps.

There are several flux enhancements over this period consistent with the traversing of a large active region across the solar disk. There appear to be inconsistencies in the data as there are drops in lower energy particles coincident with increases in higher energy particles on the 4th August and an enhancement on the 5th August after the first peak which is possibly a data error which could not be easily corrected for. Considering that the channels in the plot are integral in energy, the data indicate that the differential proton flux in the $10-30 \mathrm{MeV}$ range dropped below that in the $30-60 \mathrm{MeV}$ range for a period of $3 \mathrm{~h}$. Although such an inversion does occur for short time periods at the onset of very well-connected events, the timing and duration of this inversion seems highly improbable.

In either case, it appears likely that there are data caveats which have not been accounted for such as pulse pile-up whereby the time integration of the instrument is too high allowing for multiple particles to contribute to the energy deposited in the detector such that multiple lower energy particles are read as a single higher energy particle, an effect which in some later instruments would be mitigated. It is challenging to determine all of the data caveats present and other data with which to perform a comparison are not available. The fluences from this time series are recorded in Table 1 . Note that the fluence values for the $>10 \mathrm{MeV}$ and $>30 \mathrm{MeV}$ protons are $78 \%$ and $68 \%$ higher than those of $1.1 \times 10^{10}$ and $5.0 \times 10^{9} \mathrm{~cm}^{2}$ reported by Shea \& Smart (1990). The values given here for $>10 \mathrm{MeV},>30 \mathrm{MeV}$ and $>60 \mathrm{MeV}$ are in good agreement with those published by King (1974).

$\mathrm{Wu}$ et al. (2009) demonstrate that were an extended EVA taking place during an SPE similar to that of August 1972 then the current 30-day exposure limit of $0.25 \mathrm{~Gy}$-Eq for the bloodforming organs recommended by the latest NASA Standard Technical Standard NASA-STD-3001 (2007) and agreed upon 


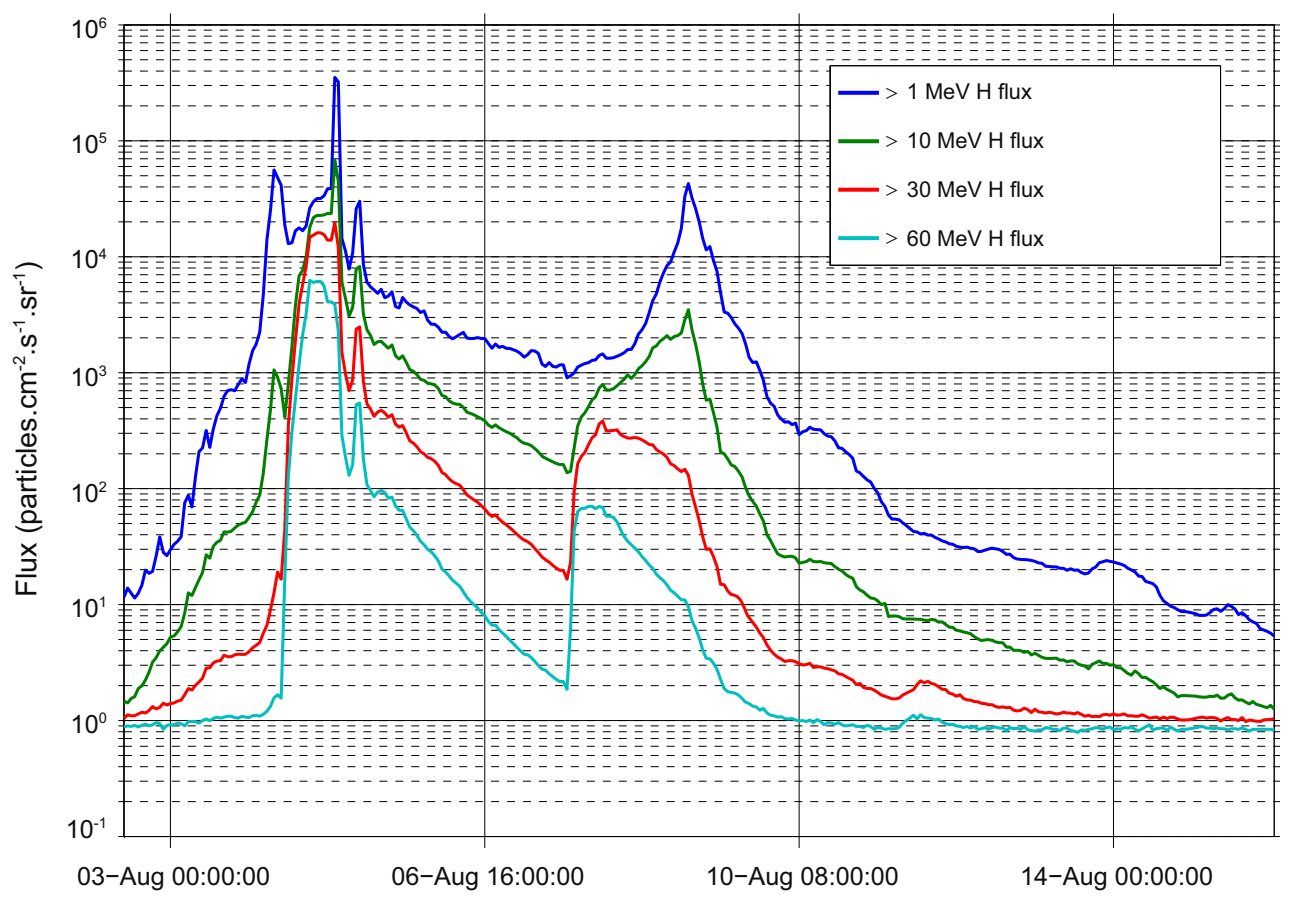

Fig. 1. Integral fluxes from the IMP-5 spacecraft measured during the large August 1972 SPE. These have been manually corrected for spikes and small data gaps have been filled using a 3rd-order polynomial.

Table 1. Fluence $\left(\mathrm{cm}^{-2}\right)$ values for the SPEs of August 1972 and October 1989. Fluxes are shown in Figures 1 and 2 respectively.

\begin{tabular}{lccc}
\hline \hline Energy $(\mathrm{MeV})$ & Aug. 1972 (from data) & Aug. 1972 (King 1974) & Oct. 1989 (from SEPEM) \\
\hline$>1$ & $8.35 \times 10^{10}$ & - & $2.75 \times 10^{11}$ \\
$>10$ & $1.96 \times 10^{10}$ & $2.25 \times 10^{10}$ & $1.85 \times 10^{11}$ \\
$>30$ & $8.42 \times 10^{9}$ & $8.10 \times 10^{9}$ & $2.54 \times 10^{9}$ \\
$>60$ & $2.58 \times 10^{9}$ & $2.45 \times 10^{9}$ & $6.72 \times 10^{8}$ \\
$>100$ & - & $5.50 \times 10^{8}$ & $2.31 \times 10^{8}$ \\
\hline
\end{tabular}

by consensus of all ISS space agencies (NASA, Roskosmos, ESA, JAXA, CSA) (Straube et al. 2010) would be exceeded. Kim et al. (2006) demonstrate that the increase in cancer risk during lifetime arising from such an event were the astronauts not behind shielding of $>2 \mathrm{~g} \mathrm{~cm}^{-2}$ would exceed the recommended 3\% level for astronauts of 25 years in age. This would clearly constitute a significant increase in risk for all astronauts given a low level of shielding and an unacceptable risk if they were on prolonged EVA.

\subsection{October 1989}

The very large SPE that occurred in October 1989 is the largest event which has been well recorded by various space-based sensors such as the Cosmic Ray Nuclear Composition (CRNC) instrument from the universities of Chicago and New Hampshire, the Charged Particle Measurements Experiment (CPME) and the Goddard Medium Energy (GME) instrument on IMP-8, and the Space Environment Monitor (SEM) on NOAA's GOES-7 spacecraft.

Processed differential proton flux time series data for the October 1989 SPE are shown in Figure 2. In the $>30 \mathrm{MeV}$ energy range it is estimated by Wu et al. (2009) that the October 1989 SPE was of similar magnitude to the SPE of August 1972 $\left(4.23 \times 10^{9}\right.$ compared to $\left.5.0 \times 10^{9}\right)$. However, from the data shown in Table 1, the August 1972 fluence is higher and the October 1989 SPE fluence lower than the fluences given $\mathrm{Wu}$ et al. (2009) resulting in a factor 3 difference. The disagreement in the October 1989 fluences can be explained by the cross-calibration of the GOES/SEM data with the IMP-8 GME data that has been performed (see the SEPEM help pages for details). Of course no such cross-calibration was possible for the August 1972 SPE.

It should be noted that the SPE of September 1989 originating from a CME produced by the same active region on the previous solar rotation had a harder spectrum than the event of October 1989 and would therefore have been of greater concern for spacecraft with higher shielding levels.

\subsection{October-November 2003}

In October-November 2003 the near-Earth environment was impacted by an SPE known as "The Halloween event". This was, by many measures, the largest SPE of solar cycle 23 with proton flux levels over $10^{5}$ times that of the background level in the $\sim 10 \mathrm{MeV}$ range and increased levels seen for approximately 2 weeks before returning to the background level. The differential proton fluxes as seen by the SEM instrument on the GOES11 spacecraft (calibrated using the IMP-8/GME data) are shown in Figure 3 for energies between 5 and $200 \mathrm{MeV}$. 


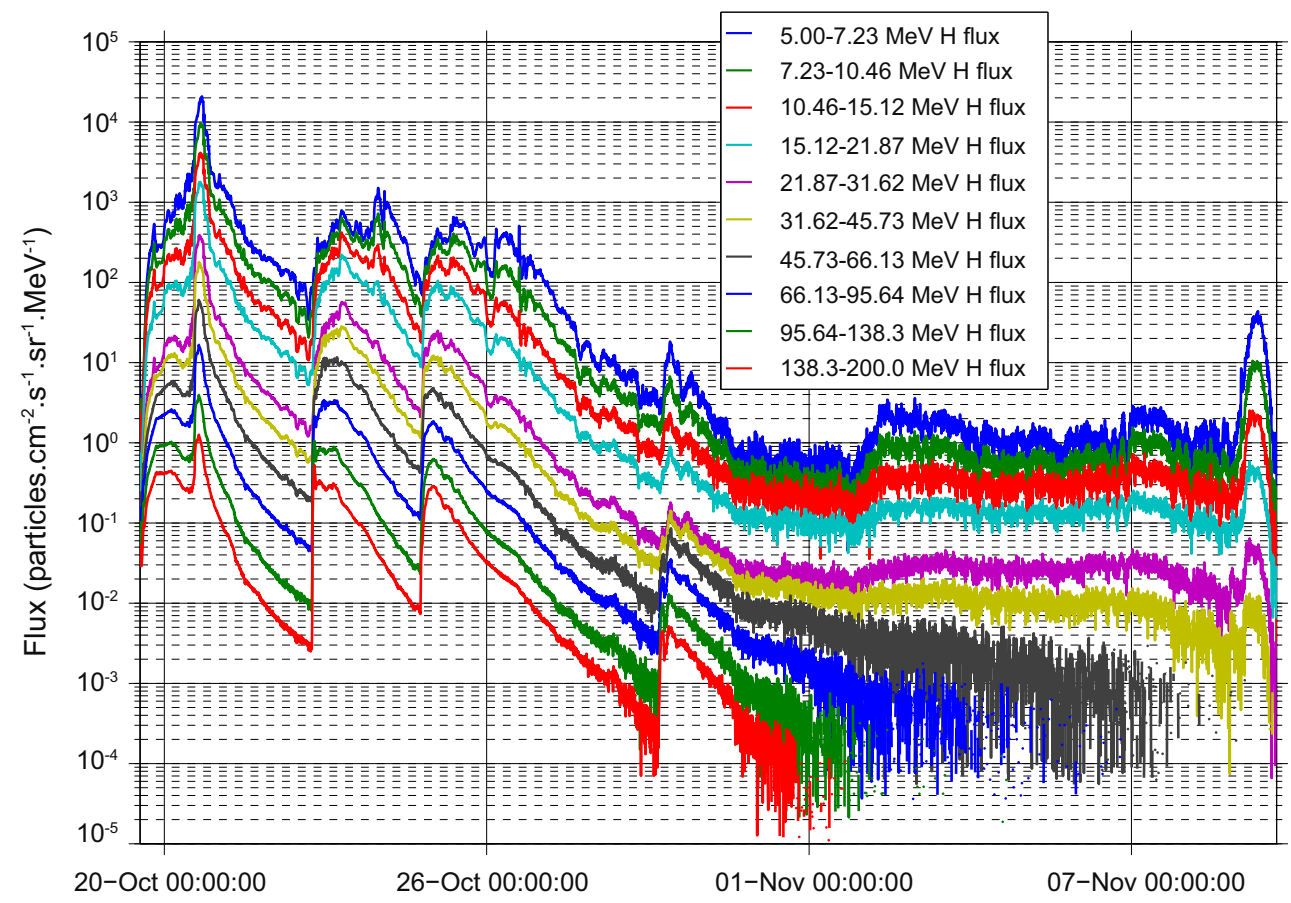

Fig. 2. Differential proton fluxes for October 1989 SPE taken from the SEPEM reference event list.

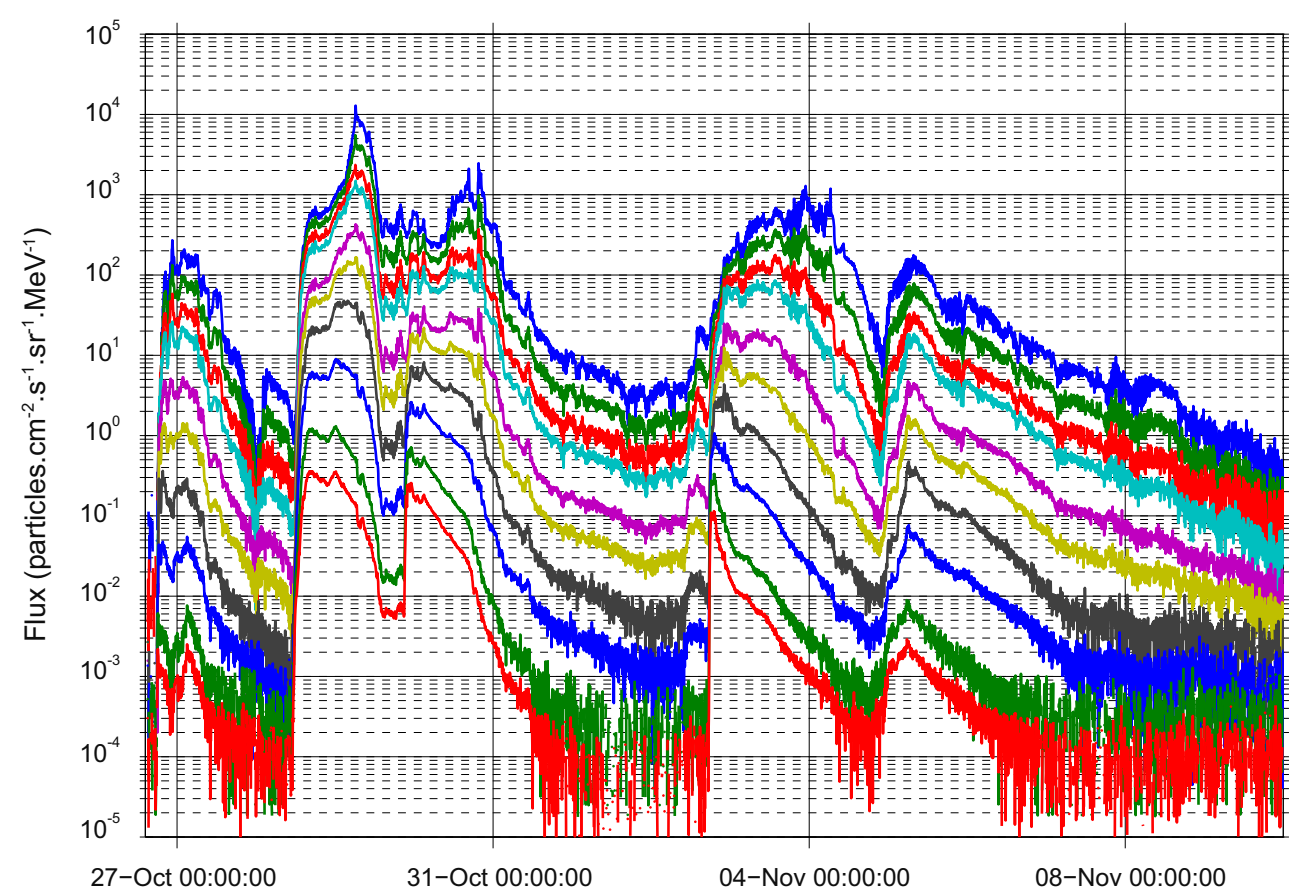

Fig. 3. Differential proton fluxes for the October-November 2003 "Halloween" SPE taken from the SEPEM reference event list. Proton energy channels are the same as those for Figure 2.

This SPE also provides a visual illustration of the radiation impacts on spacecraft instruments through use of the $\mathrm{SOHO} /$ LASCO (C3) coronagraph. The LASCO instrument has been observing the solar corona and recording CMEs since its launch in December 1995. The largest solar proton enhancement occurred on the 28th October following an X17.2 class flare. The SOHO/LASCO 149 CME Catalogue notes that LASCO and EIT (EUV imager on-board SOHO) observed a CME on
2003/10/28 which was first observed in C2 (medium-angle coronagraph) at 10:54 UT. It had developed into a full halo CME in C2 by 11:30 UT and appears in C3 (wide-angle coronagraph) images by 11:42. The CME had a speed of $2500 \mathrm{~km} \mathrm{~s}^{-1}$, the active region was close to the centre of the solar disk. Figure 4 shows the emergence of the $\mathrm{CME}$ as observed by LASCO C3 from 11:42 UT to 12:42 UT. In the last image a large amount of "snow" is seen on the detector 


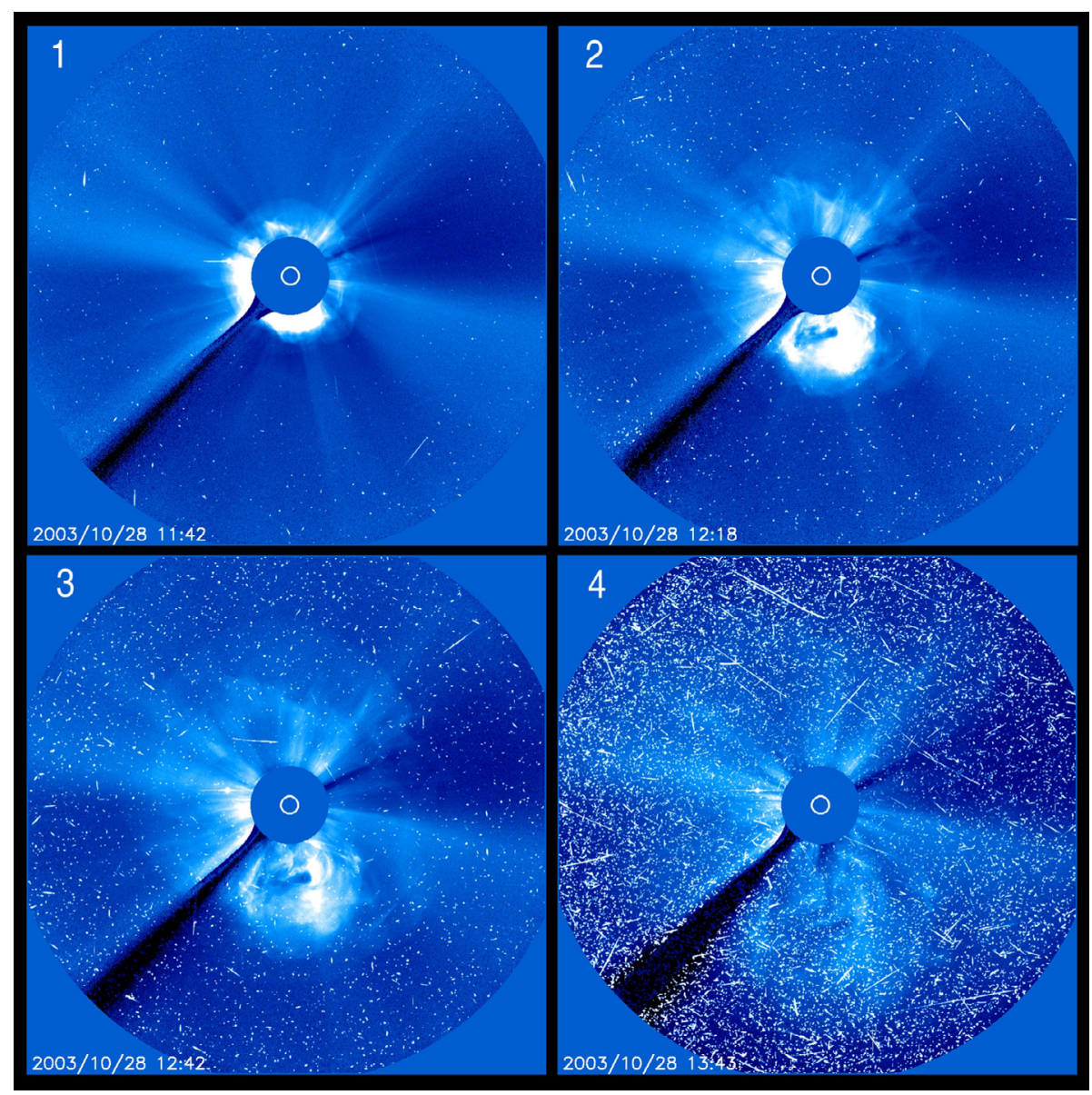

Fig. 4. SOHO LASCO C3 images from 28th October 2003 illustrating "snow" on the detector resulting from high energy protons accelerated by a fast CME and constituting part of the well-known "Halloween" SPE [Credit: ESA/NASA/NRL].

obscuring the image, this is caused by particle radiation hits resulting from the increase in the high energy protons shown in Figure 3. This event is studied in detail by Mewaldt et al. (2005).

\subsection{July 2012 (STEREO-A)}

On 23rd July 2012 the SECCHI instrument on-board the STEREO-A spacecraft imaged an extremely fast CME which appeared as a halo CME from the spacecraft's vantage point indicating that the spacecraft would feel the full impact of the particle radiation storm. Two images separated by $1 \mathrm{~h}$ show the evolution of this CME from the STEREO-A perspective (Fig. 5). The NASA Goddard Space Weather Research Center reports that there was an increase of over five orders of magnitude in protons in the $13-100 \mathrm{MeV}$ range at the $\mathrm{CME}$ arrival observed by the High Energy Telescope (HET) in the IMPACT suite on-board STEREO-A and that considering its arrival time, the initial speed of CME could be as high as $3400 \mathrm{~km} \mathrm{~s}^{-1}$. The HET proton fluxes are shown in Figure 6. The movie available on the Naval Research Laboratory website shows levels of "snow" resulting from the increase in high energy protons. The blinding of the detector is similar, if not more severe, than the blinding of the LASCO instrument onboard by the Halloween SPE shown in Figure 4.

As a result of the CME speed and direction, it was thought that this SPE could be of similar magnitude to the famous Carrington event of 1859. In order to compare the data from this event to data from the October 1989 event the flux for each time step in Figure 6 has been re-binned into the energies evenly separated on a logarithmic scale as was done on the SEPEM system by assuming a power law fit to flux as a function of energy between the two nearest energy bins for each of the target channels. Figure 7 shows the result of this method applied to the total event fluence to illustrate the procedure.

Figure 8 shows the proton fluxes for the first 3 days of the October 1989 SPE on the left-hand side and the interpolated fluxes on the right-hand pane for the first 3 days of the July 2012 SPE observed by STEREO-A for the six energy channels that lie within the IMPACT/HET energy range. The temporal and flux scales are identical. These two events appear similar albeit there are small differences in the onset and peak values with the July 2012 SPE having a more rapid onset and higher peak flux values and a sharper decay. This may indicate that the July 2012 SPE was better connected to the observer than the October 1989 even though both originated from central solar events. As the July 2012 SPE was not followed by further CME-driven shocks the overall fluence for the event was less than that for the SPEs of both October 1989 and October 2003 for the energies over which the fluxes were measured by the IMPACT instrument.

\subsection{The Carrington event}

The so-called "Carrington event" was a solar flare observed by the British astronomer Richard Carrington on 1st Septmeber 


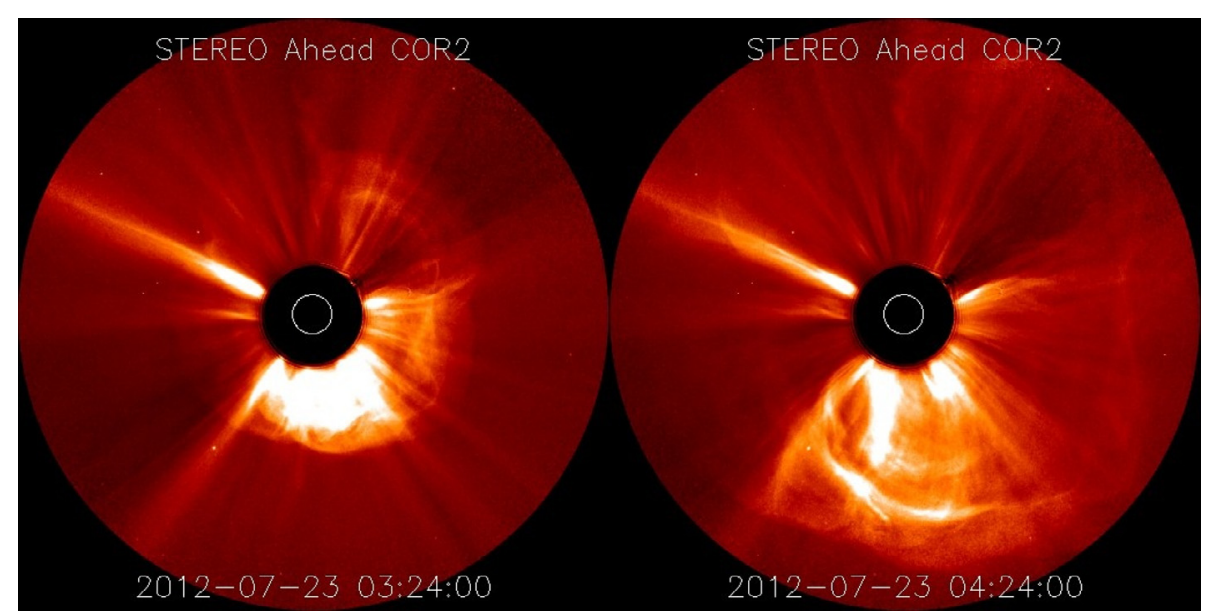

Fig. 5. SECCHI COR 2 coronagraph images from 23rd July 2012 showing the extremely fast $\sim 3400 \mathrm{~km} \mathrm{~s}^{-1}$ CME as seen by STEREO-A [Credit: NASA].

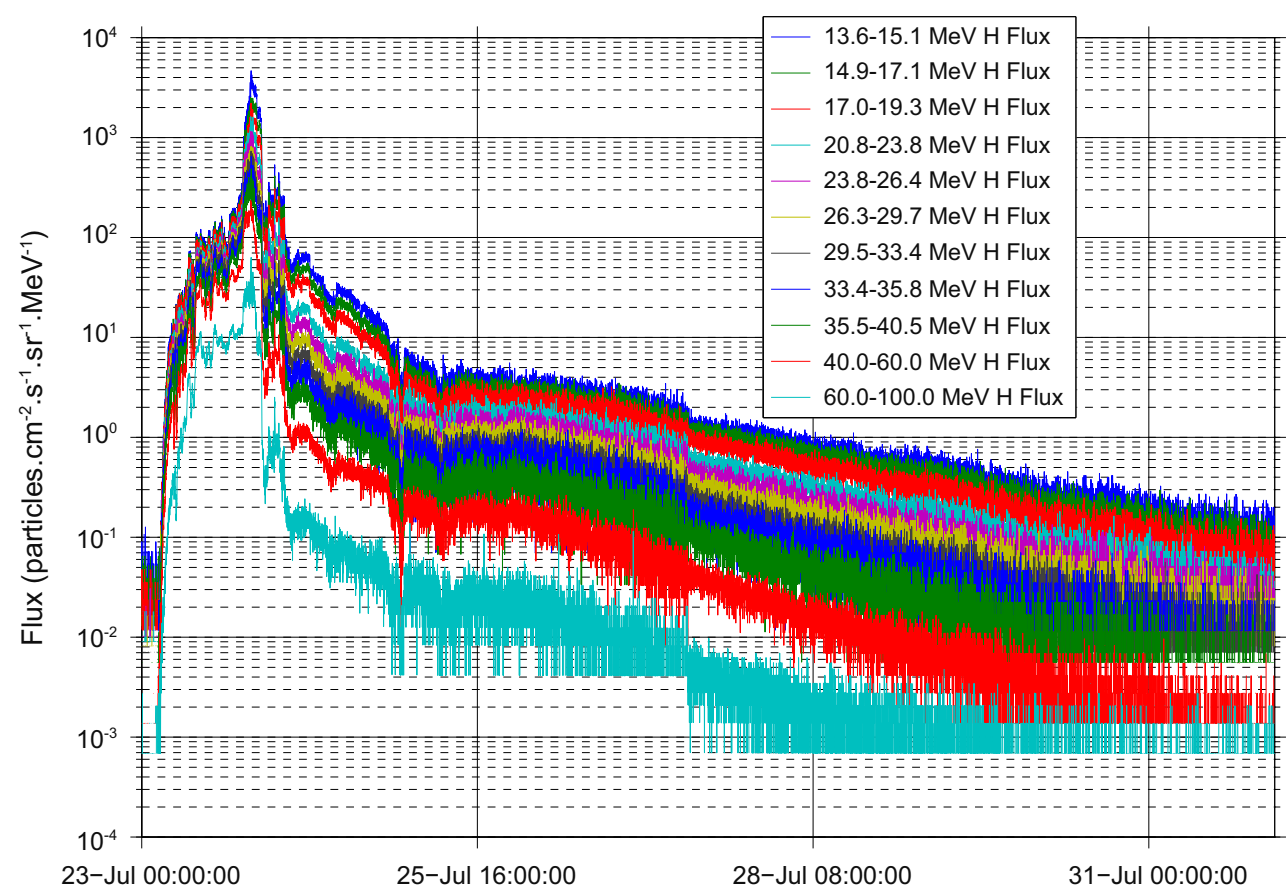

Fig. 6. Differential proton fluxes for the July 2012 SPE observed by the IMPACT/HET instrument on-board STEREO-A.

1859 (Carrington 1860). The DST (Disturbance storm time index), a measure of the change in the Earth's magnetic field at the Earth's equator averaged over $1 \mathrm{~h}$, is estimated to have dropped to $-850 \mathrm{nT}$ (Siscoe et al. 2006) - well in excess of the deviation resulting from the magnetic storm of March 1989 which caused widespread blackouts in Quebec for approximately $6 \mathrm{~h}$. In 1859, auroras were seen as far south as Florida in the United States (Clauer \& Siscoe 2006).

The associated CME is believed to have had a very fast transit time of $17.5 \mathrm{~h}$ before its arrival at the Earth. Work by McCracken et al. (2001) deduced estimates for the $>30 \mathrm{MeV}$ proton fluence for the resulting SPE by relation to the nitrate levels found in ice cores. Later, Smart et al. (2006) presented a possible proton intensity-time profile for the SPE. However, this work has recently been questioned by Wolff et al. (2012) who conclude that, although there would undoubtedly have been some nitrate deposition as a result of generation in the stratosphere, the signature of the 1859 SPE did not leave an observable imprint in nitrate in ice. The authors report that it is overwhelmingly likely that the nitrate deposition found in the Greenland ice core resulted from a biomass burning plume. This is not to say that there was not an extreme SPE associated with the Carrington Flare but that it may not be possible to determine its magnitude. In the absence of data for any such extreme SPEs, herein the value reported by McCracken et al. is used in conjunction with the spectral shape of the March 1991 SPE as reported by Townsend et al. (2003) to compare it to model outputs. Townsend et al. also produced a spectrum based on the September 1989 SPE but this was more of a Western event than a central meridian event so it was decided to use the spectral shape from March 1991. It is interesting to note that the authors projected the dose in Si for the SPE behind various thicknesses of $\mathrm{Al}$ shielding and found values which exceed the $100 \%$ limits proposed by Xapsos et al. (2000). 
P. Jiggens et al.: The magnitude and effects of extreme solor particle events

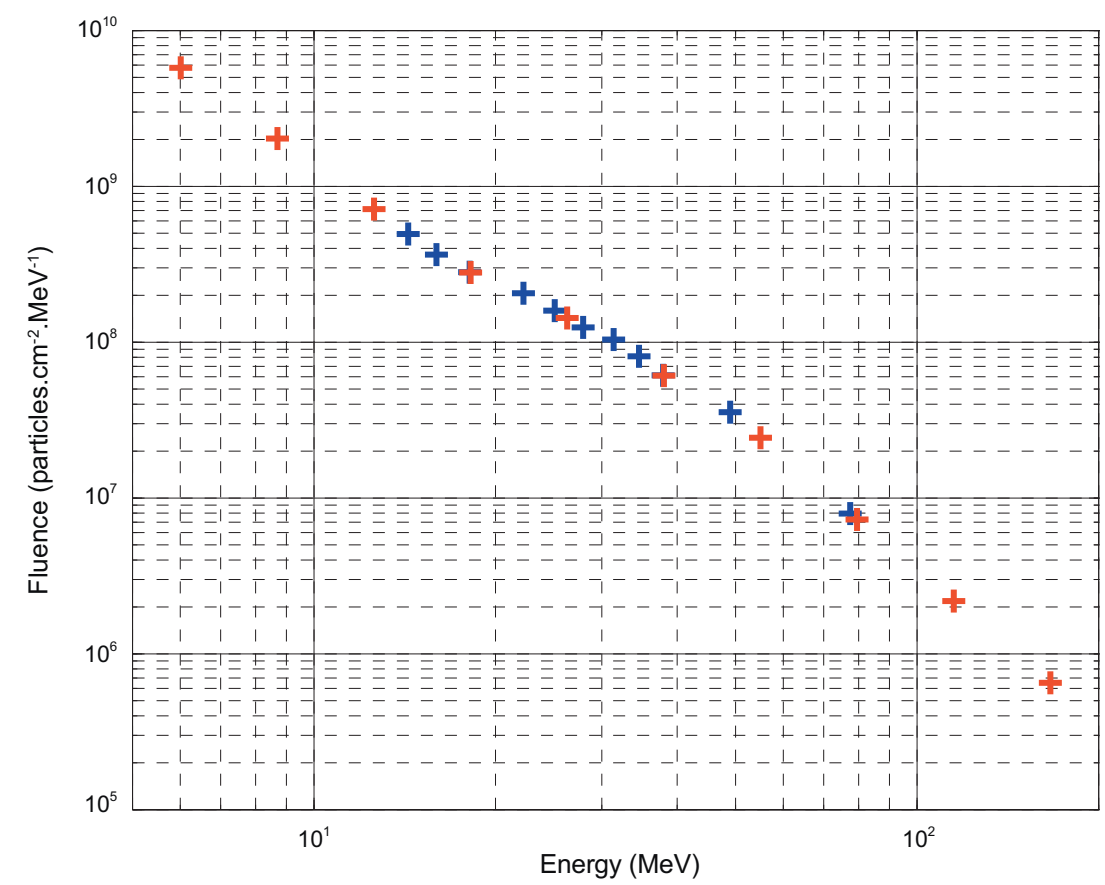

Fig. 7. Illustration of the procedure used to re-bin data for the SPE of July 2012 observed by the IMPACT/HET instrument on-board STEREOA. The data in this example are the total event fluence. The raw data are given by the blue crosses and the interpolated/extrapolated data are given by the red crosses.

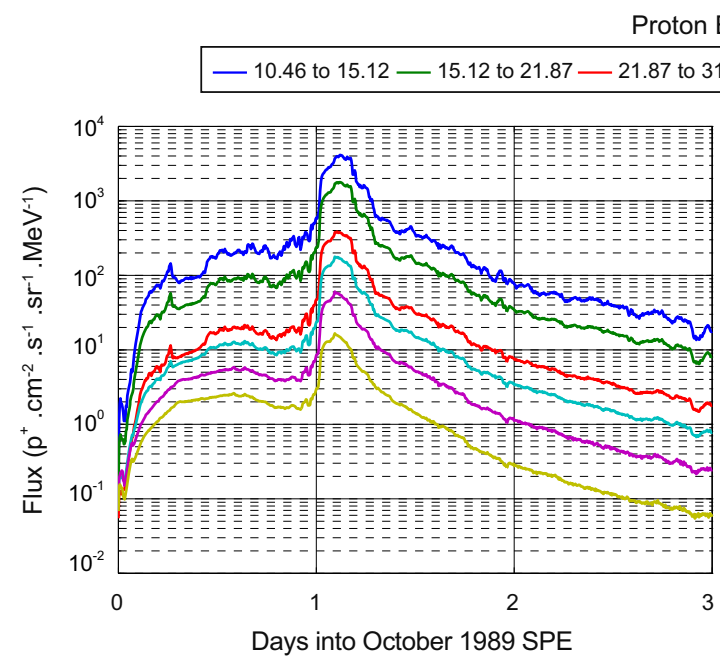

Proton Energy (MeV)

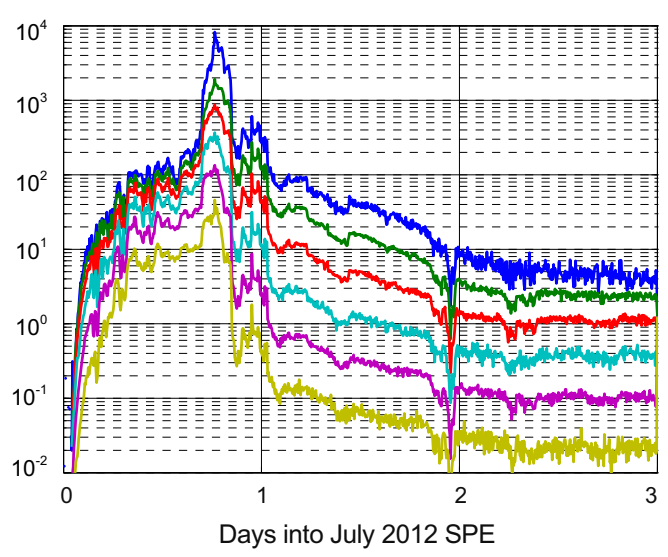

Fig. 8. Comparison between the flux profiles of the October 1989 SPE (left) and the July 2012 SPE (right) for the first 3 days of the events.

\subsection{Modelled spectra}

Figure 9 shows outputs from the SEPEM model (Jiggens et al. 2012) and event spectra for the August 1972, October 2003, July 2012 (STEREO-A) and the estimate for the Carrington event (Townsend et al. 2003). The data for the historical SPEs do not include data ingested into the SEPEM model as the purpose here is not to validate the modelling methodology but simply to allow comparison. For the August 1972 SPE the raw IMP-5 data (illustrated in Fig. 1) were used, for the July 2012 SPE the STEREO-A/HET data (illustrated in Fig. 6) were used while the October 2003 SPE is shown using spectral fits provided by Mewaldt et al. (2005), this last event is included in the SEPEM database but the source data and processing are different.
The objectives of this study are the assessment of radiation effects from SPEs on a nominal manned mission of 9 months duration at solar maximum and the possible radiation impacts of a worst-case extreme SPE. SPE spectra were estimated using models presented by Jiggens et al. (2012) and implemented in the SEPEM framework. A spectrum was produced corresponding to a $95 \%$ confidence level ( $95 \%$ confidence of not being exceeded by any SPE at any energy) for a 9-month mission at solar maximum along with a more extreme event using a $99 \%$ confidence level. In addition, to produce a worst-case event the model was run for a mission duration of 7 years, considered to be the average duration of a solar maximum period, with a $99.9 \%$ confidence level with the resulting curve in Figure 9 labelled "SEPEM Extreme SPE". This spectrum was below the estimation for the Carrington event presented 


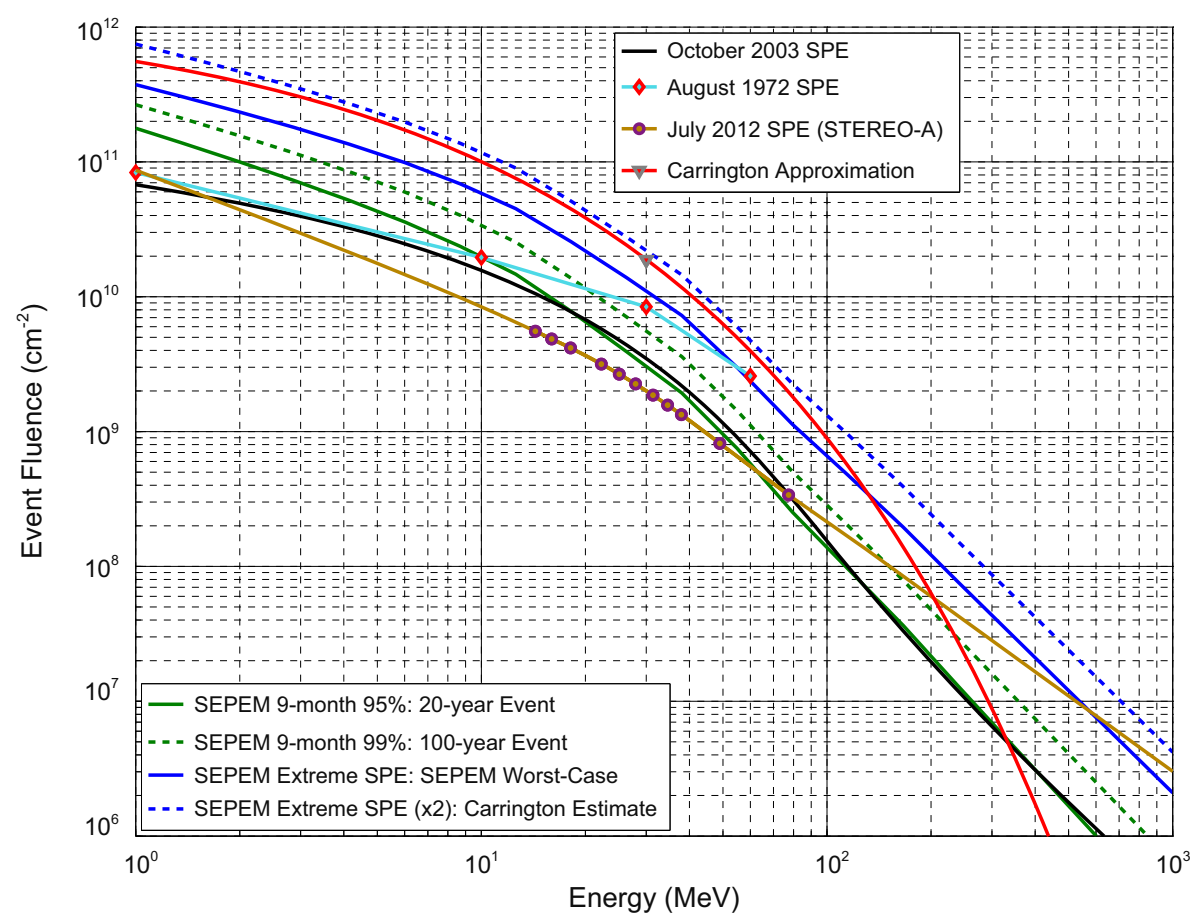

Fig. 9. Fluence Spectra of four events produced using the SEPEM statistical model compared to some well-known large SPEs from the space age and the estimate of the Carrington event spectra based on nitrate data from ice cores and the spectral shape of the 23rd March 1991 SPE published by Townsend et al. (2003).

by Townsend et al. (2003) and was therefore multiplied by two to produce a further curve labelled "SEPEM Extreme SPE $(\times 2)$ " which more closely matched that estimation.

From Figure 9 it can be seen that the spectra of the SEPEM $95 \%$ confidence level SPE for a mission of 9 months closely agrees with that of the large October 2003 SPE, the largest SPE of the past 20 years in the 10 s of MeV energy range. In order to estimate the probability of this modelled SPE occurring in a 20 -year period a statistical extrapolation can be performed assuming that the duration of a solar cycle is 11 years with 4 quiet years producing negligible probability of a severe SPE compared to the 7 active years:

Probability of exceeding $=$

$$
\begin{aligned}
& 1 \text { - probability of not exceeding } \\
& \qquad=1-0.95\left(\frac{20}{0.75} \times \frac{7}{\Pi 1}\right)=0.58 .
\end{aligned}
$$

This indicates that there is a $58 \%$ probability that at least one SPE will exceed this fluence boundary at any given energy. Although there remains a $42 \%$ chance that no such SPE will occur there is also a non-negligible chance that more than one SPE might exceed the given fluence. Although any assessment of the mean rate of occurrence will not be exact due to the uncertainties connected to variations in solar cycle activity and duration, if extreme events are considered to follow a Poisson distribution the mean event occurrence corresponding to a $42 \%$ chance of no sufficiently large event occurring is 0.87 events for a given time period. As this is of the order of unity, this modelled curve can be taken to be approximately a 1-in-20-year SPE. Similarly, the result for a $99 \%$ confidence level SPE, appropriate for the July 2012 STEREO-A event for energies between 100$200 \mathrm{MeV}$, for a mission of 9 months can be extrapolated to a 100 -year probability giving a similar result:

$$
\text { Probability of exceeding }=1-0.99^{\left(\frac{100}{0.75} \times \frac{7}{11}\right)}=0.57 \text {. }
$$

Lastly, the result for a $99.9 \%$ confidence level SPE for a mission of 7 years can extrapolated to a 10,000-year probability:

$$
\text { Probability of exceeding }=1-0.999^{\left(\frac{10000}{7} \times \frac{7}{11}\right)}=0.60 \text {. }
$$

The labels of "20-year Event" and "100-year Event" are used in following sections wherein the determination of the likely effects of very large and extreme SPEs are calculated as they are more concise and their meaning is more easily understandable by a broader audience. Based on current statistics the "SEPEM Extreme SPE" is likely to occur approximately once in 10,000 years and the probability of the SEPEM Extreme SPE $(\times 2)$ event based on the present dataset is negligibly small. However, the limitation in terms of time-span of the dataset (only four solar cycles) means that the part of the distribution corresponding to such a low likelihood of being exceeded is very poorly resolved (see Rosenqvist and Hilgers 2003) and therefore hereafter they are labelled simply as "SEPEM Worst-Case" and "Carrington Estimate" respectively.

The statistical modelling resulting in the fluences given in Figure 9 has been done separately for 10 energy channels in the 5-200 MeV range (with two power law extrapolations beyond that range) and although clearly there is a correlation in fluxes at different energies during SPEs the spectra do vary significantly in different events. For this reason, in the following three sections the spectra are input into the effects tools in the standard way (after the statistical analysis) but also events are modelled statistically based on a derived effects time series using the SEPEM system whereby the energies are combined to determine the effect point-by-point in time prior to the statistical analysis. This second method is designed to avoid issues of possible overestimation (conservatism) through the multiplication of confidence levels. 
P. Jiggens et al.: The magnitude and effects of extreme solor particle events

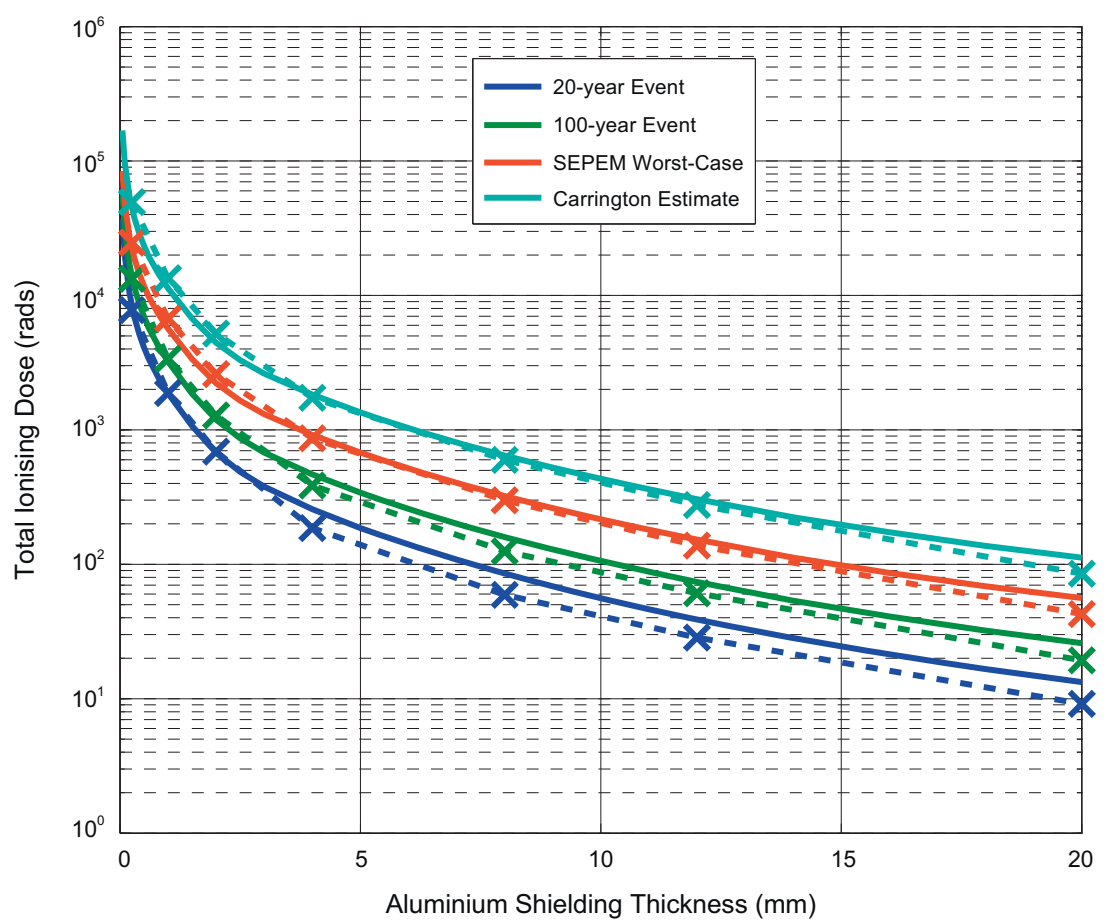

Fig. 10. Plots of total ionising dose (TID) against shielding thickness for for the four SPEs shown in Figure 9: SPENVIS method (solid line); SEPEM method (dashed line with crosses).

Table 2. Total ionising dose (TID) values calculated using SPENVIS tools and the SEPEM system for the four SPEs shown in Figure 9 in units of rads.

\begin{tabular}{|c|c|c|c|c|c|c|c|c|}
\hline \multirow[t]{2}{*}{$\mathrm{Al}(\mathrm{mm})$} & \multicolumn{2}{|c|}{ 20-year event } & \multicolumn{2}{|c|}{ 100-year event } & \multicolumn{2}{|c|}{ SEPEM W-C } & \multicolumn{2}{|c|}{ Carrington est. } \\
\hline & SPEN & SEPEM & SPEN & SEPEM & SPEN & SEPEM & SPEN & SEPEM \\
\hline 1.0 & $1.87 \times 10^{3}$ & $1.88 \times 10^{3}$ & $3.22 \times 10^{3}$ & $3.38 \times 10^{3}$ & $5.66 \times 10^{3}$ & $6.72 \times 10^{3}$ & $1.13 \times 10^{4}$ & $1.34 \times 10^{4}$ \\
\hline 2.0 & $6.75 \times 10^{2}$ & $6.89 \times 10^{2}$ & $1.19 \times 10^{3}$ & $1.28 \times 10^{3}$ & $2.22 \times 10^{3}$ & $2.60 \times 10^{3}$ & $4.45 \times 10^{3}$ & $5.20 \times 10^{3}$ \\
\hline 4.0 & $2.57 \times 10^{2}$ & $1.86 \times 10^{2}$ & $4.67 \times 10^{3}$ & $3.86 \times 10^{2}$ & $9.16 \times 10^{2}$ & $8.66 \times 10^{2}$ & $1.83 \times 10^{3}$ & $1.73 \times 10^{3}$ \\
\hline 8.0 & $8.53 \times 10^{1}$ & $5.93 \times 10^{1}$ & $1.59 \times 10^{2}$ & $1.25 \times 10^{2}$ & $3.22 \times 10^{2}$ & $2.99 \times 10^{2}$ & $6.45 \times 10^{2}$ & $5.98 \times 10^{2}$ \\
\hline 12 & $3.91 \times 10^{1}$ & $2.85 \times 10^{1}$ & $7.39 \times 10^{1}$ & $6.09 \times 10^{1}$ & $1.53 \times 10^{2}$ & $1.38 \times 10^{2}$ & $3.05 \times 10^{2}$ & $2.76 \times 10^{2}$ \\
\hline 20 & $1.33 \times 10^{1}$ & $9.16 \times 10^{0}$ & $2.59 \times 10^{1}$ & $1.93 \times 10^{1}$ & $5.61 \times 10^{1}$ & $4.26 \times 10^{1}$ & $1.12 \times 10^{2}$ & $8.52 \times 10^{1}$ \\
\hline
\end{tabular}

\section{Total ionising dose effects}

The four SPE spectra produced by the environment model as described in Section 2.6 were input into a variety of effects tools to determine how damaging events of these sizes would be. The first of these was an estimation of the TID behind a layer of aluminium shielding of various thicknesses. TID is a measure of cumulative long-term ionising radiation damage which can induce a degradation of electrical and functional properties and can lead to the permanent failure of an electronic component.

The traditional method for determining TID is to input the spectra into an effects tool. In this case SHIELDOSE-2 (Seltzer 1994) was used to produce the solid lines in Figure 10 for TID as a result of each simulated SEP environment for shielding thicknesses from 0 to $20 \mathrm{~mm}$ (aluminium). This is labelled the SPENVIS method (as it is the way in which this tool is presently implemented in SPENVIS). In addition, using SEPEM, MULASSIS (Lei et al. 2002) was run point-by-point on the instantaneous spectra combining 10 energy channels of flux time series data for 7 aluminium slab shielding thicknesses. The result of this was a response function giving a dose for each point in time, i.e., a new time series with ionising dose deposited in the component per time step rather than particle flux. The statistics are then run directly on the TID time series by defining dose distributions and event thresholds (in Rads) in the same way that one would usually do in order to create a particle fluence model based on the flux time series. The advantage of this method is that the focus is placed on the points which are significant for the specific effect and component/shielding configuration.

As shown in Figure 10 and tabulated in Table 2, the results from the SEPEM-MULASSIS method compare well to those from the SPENVIS method. Minor differences at higher shielding thicknesses may result from the exclusion in SEPEM of protons with energies greater than $200 \mathrm{MeV}$. Although the energy required to penetrate this thickness for a normally incident proton is only $70 \mathrm{MeV}$ and fluxes drop by an order of magnitude between 70 and $200 \mathrm{MeV}$ for an average SPE, the penetration energy for obliquely incident particles would be significantly higher. The larger differences seen especially in the 20-year event may result from the summation of $95 \%$ confidence runs for a 9-month time period at different energies which will tend to overpredict the true 95\% SPE fluence 


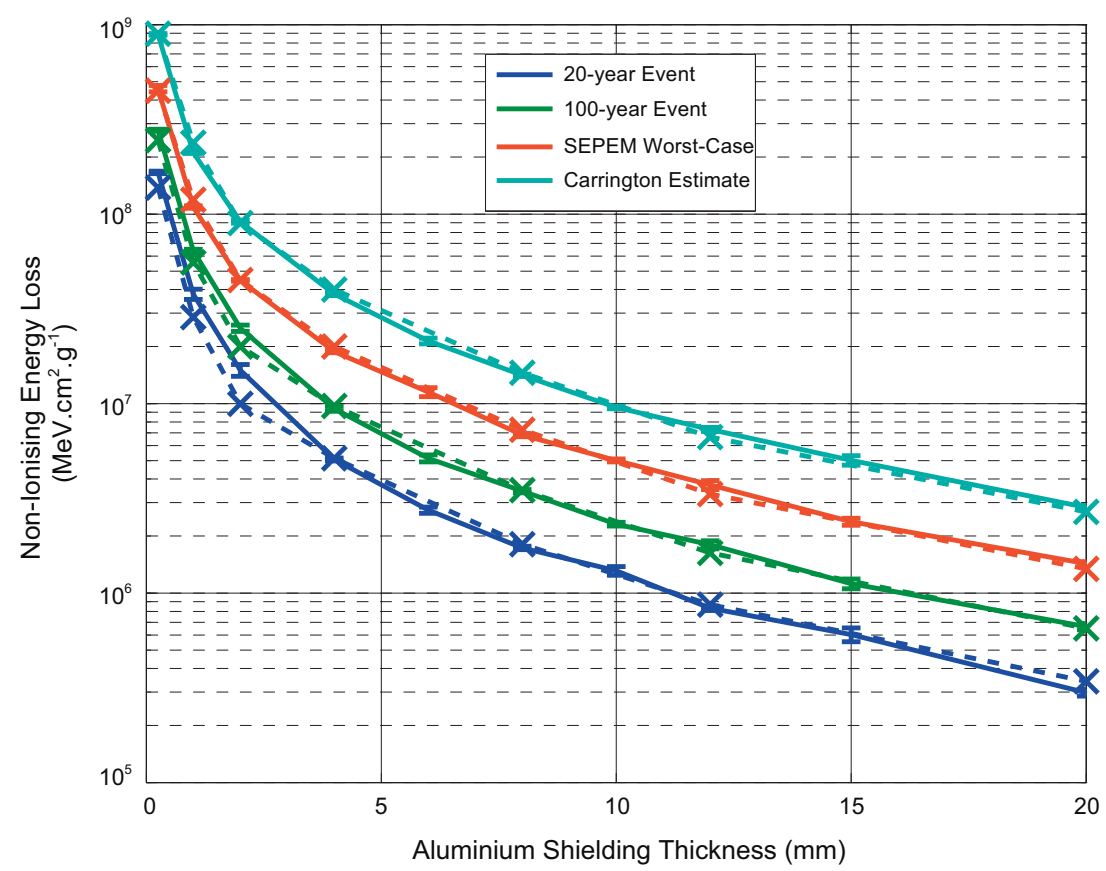

Fig. 11. Plots of non-ionising energy loss (NIEL) against shielding thickness for for the four SPEs shown in Figure 9: SPENVIS method (Solid line); SEPEM method (dashed line with crosses).

Table 3. Non-ionising energy loss (NIEL) values calculated using SPENVIS tools and the SEPEM system for the four SPEs shown in Figure 9 in units of $\left(\mathrm{MeV} \mathrm{cm} \mathrm{g}^{-1}\right)$.

\begin{tabular}{|c|c|c|c|c|c|c|c|c|}
\hline \multirow[t]{2}{*}{$\mathrm{Al}(\mathrm{mm})$} & \multicolumn{2}{|c|}{ 20-year event } & \multicolumn{2}{|c|}{ 100-year event } & \multicolumn{2}{|c|}{ SEPEM W-C } & \multicolumn{2}{|c|}{ Carrington est. } \\
\hline & SPEN & SEPEM & SPEN & SEPEM & SPEN & SEPEM & SPEN & SEPEM \\
\hline 1.0 & $3.78 \times 10^{7}$ & $2.86 \times 10^{7}$ & $6.41 \times 10^{7}$ & $5.55 \times 10^{7}$ & $1.09 \times 10^{8}$ & $1.19 \times 10^{8}$ & $2.11 \times 10^{8}$ & $2.38 \times 10^{8}$ \\
\hline 2.0 & $1.50 \times 10^{7}$ & $9.98 \times 10^{6}$ & $2.50 \times 10^{7}$ & $2.00 \times 10^{7}$ & $4.47 \times 10^{7}$ & $4.49 \times 10^{7}$ & $9.11 \times 10^{7}$ & $8.98 \times 10^{7}$ \\
\hline 4.0 & $5.07 \times 10^{6}$ & $5.14 \times 10^{6}$ & $9.38 \times 10^{6}$ & $9.72 \times 10^{6}$ & $1.88 \times 10^{7}$ & $2.00 \times 10^{7}$ & $3.79 \times 10^{7}$ & $4.00 \times 10^{7}$ \\
\hline 8.0 & $1.73 \times 10^{6}$ & $1.81 \times 10^{6}$ & $3.45 \times 10^{6}$ & $3.49 \times 10^{6}$ & $6.82 \times 10^{6}$ & $7.27 \times 10^{6}$ & $1.41 \times 10^{7}$ & $1.45 \times 10^{7}$ \\
\hline 12 & $8.32 \times 10^{5}$ & $8.69 \times 10^{5}$ & $1.80 \times 10^{6}$ & $1.63 \times 10^{6}$ & $3.20 \times 10^{6}$ & $3.33 \times 10^{6}$ & $7.30 \times 10^{6}$ & $6.66 \times 10^{6}$ \\
\hline 20 & $2.98 \times 10^{5}$ & $3.42 \times 10^{5}$ & $6.65 \times 10^{5}$ & $6.51 \times 10^{5}$ & $1.43 \times 10^{6}$ & $1.34 \times 10^{6}$ & $4.13 \times 10^{6}$ & $2.68 \times 10^{6}$ \\
\hline
\end{tabular}

spectra. The doses resulting from any of these SPEs would not be particularly challenging for space electronic components behind a typical spacecraft shielding but behind very thin shielding could provide a significant contribution to the mission TID.

\section{Displacement damage effects}

NIEL describes the rate of energy loss due to atomic displacements and photon emission as particles traverse a material, often known as material degradation or bulk damage. NIEL effects are important for semiconductor electronic components, solar arrays, but also for imaging devices, such as CCDs and CMOS active pixel sensors, which show dark current spikes due to displacement damage.

As for the TID analysis two modelling methods were compared for the NIEL analysis for the large/extreme SPEs shown in Figure 9. The standard method gives the displacement damage energy deposition per unit mass of material which is obtained directly from the convolution of transmitted particle fluence with NIEL coefficients (once again this is labelled the "SPENVIS method"). The alternative approach was to run the statistical models directly on the NIEL time series resulting from the MULASSIS response function produced in the
SEPEM system. The NIEL curves used in both cases were those presented by Jun et al. (2003).

The results of the two methods compare very well as shown in the Figure 11. Tabulated outputs are given in Table 3. More significant differences are seen for the smaller SPEs at lower shielding thicknesses. The reason for this deviation may be due to the summation of confidences in the SPENVIS method where the results for 10 separate energy channels at (in the example of the 1-in-20year event) a 95\% confidence level are combined which may overestimate the true $95 \%$ confidence level which should be calculated through a single statistical analysis as is done for the SEPEM method. This could account for an overestimation of the $95 \%$ worst-case for NIEL for the SPENVIS method. All other results are within, or very nearly within, the error bars shown. These error bars represent the variance in the statistics for the MULASSIS/ NIEL tool on the SPENVIS method results.

These results show that significant degradation in the performances can be caused by displacement damage induced by a single harsh SPE.

\section{Single event effects (SEEs)}

Electronic components can be susceptible to single event effects (SEEs), where a single proton or heavy ion deposits sufficient 
P. Jiggens et al.: The magnitude and effects of extreme solor particle events

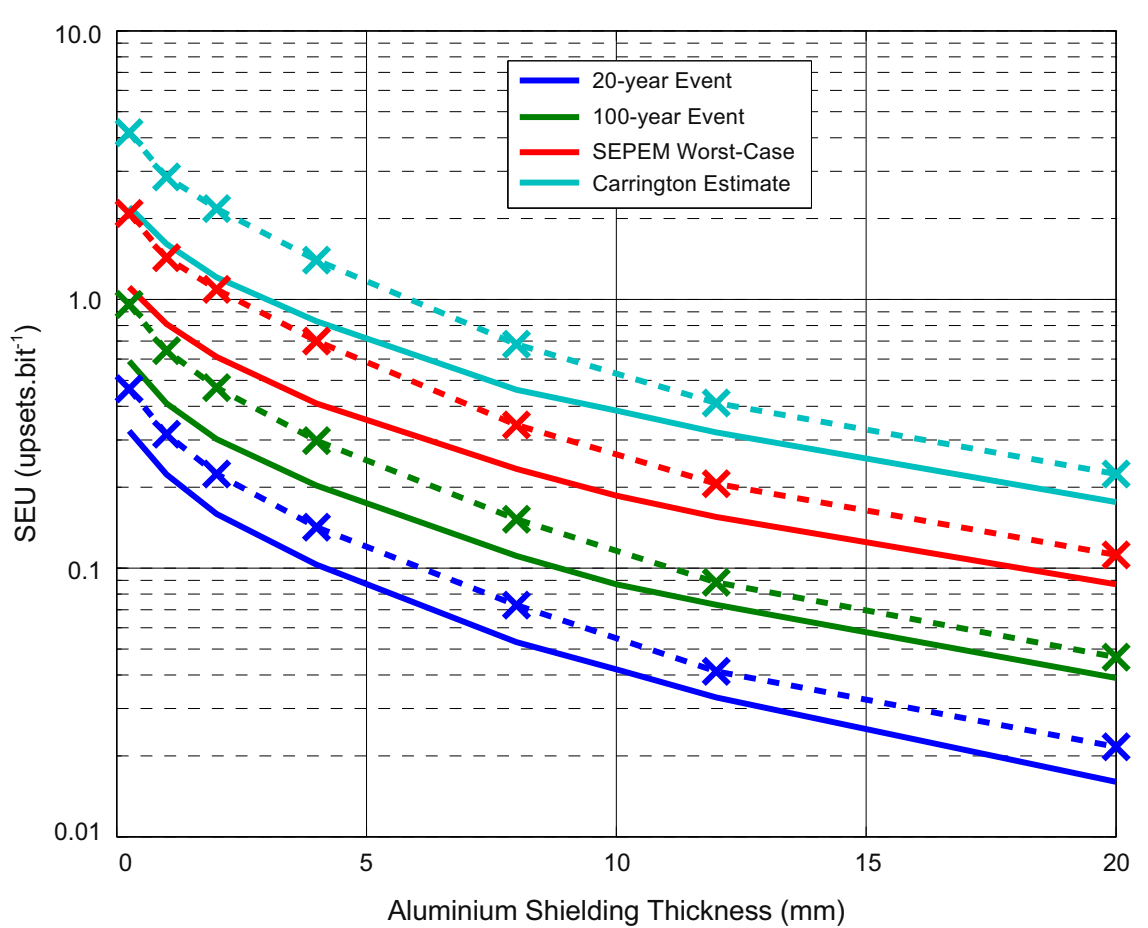

Fig. 12. Plots of single event upset (SEU) rate in Cypress 93L422AM against shielding thickness for for the four SPEs shown in Figure 9: direct method (solid line); SEPEM method (dashed line with crosses).

energy within the component as to cause a device malfunction. Such malfunctions can range from soft errors or "bit-flips", to a more catastrophic latch up event, resulting in permanent damage to the device.

Using the device proton cross-section and Bendel function for the relation between interaction cross-section and proton Linear Energy Transfer (LET) (Stapor et al. 1990) for the Cypress 93L422AM $256 \times$ 4-bit fully decoded random access memory (Petersen 1998), Figure 12 below compares the expected number of SEUs per bit over the event as a function of spherical shell shielding thickness for the SEPEM generated statistical SEU models (dashed) with those produced directly from the event fluence model (solid). Both methods use the MULASSIS tool to determine the flux behind the various layers of shielding combined with a Bendel function fit to experimental data from the example component to map the proton energy to the SEU cross section, in other words the upset rate as a function of energy. To avoid gross discrepancies only protons were used with energies up to $200 \mathrm{MeV}$.

The results are very different with the SEPEM output being far higher than the direct method. It is believed that this is due to the method of generating the response function to calculate the SEU rate time series. For this a spectral form for each bin is needed and with no knowledge of this a priori it is taken to be approximately flat. As a result the output SEU cross-section for the bin might be too high resulting in a higher SEU rate. This may be especially important for low shielding thicknesses where the majority of SEU-causing flux would come from the low energy particles where the spectrum is softer. The result of a greater proportion of particles in the lower energy bins being assumed to be of sufficient energy to penetrate has a huge impact on the total SEUs per bit.

In order to resolve this it should be possible to fit smooth spectra to the fluxes. However, such spectral forms cannot be assumed for each 5-min interval but only over the course of whole SPEs. In effect, this is the analysis which has been performed using the direct method and in the future a composite method to define events using a coarse estimation of SEU rate and a refined number of SEUs per bit calculated using a spectral fit to the fluence over the SPE will be implemented. The divergence of the curves in Figure 12 at lower shielding thicknesses maybe due to the definition of the worst-case event (correctly) including consideration of the shielding configuration by the SEPEM method which is ignored in the direct method.

Protons deposit insufficient charge per unit length to cause SEUs directly by ionisation and the primary method for protons to cause SEUs in components is through secondary particles resulting from nuclear interaction which have higher stopping power and greater potential for causing SEUs. Even then, the energy deposited by a single proton stopping in the target conductor is only capable of causing a SEU in a soft component (LET $<15 \mathrm{MeV} \mathrm{cm}^{-2} \mathrm{mg}^{-1}$ ). Clearly, the model only including the contribution of solar protons up to $200 \mathrm{MeV}$ underestimates the SEU rate where a disproportionate contribution comes from heavier ions capable of causing SEUs through direct ionisation. These heavy ions come from both solar events and from sources outside the solar system (GCRs). The best example of the inclusion of solar heavy ions in a statistical model to date is that from Xapsos et al. (2007) while widely used GCR models include those from Badhwar \& O'Neill (Badhwar \& O’Neill 1996; O'Neill 2006, 2010) and from Nymmik et al. (1996), Nymmik (1996) and the present ISO standard (15390, 2004).

Work is progressing to extend the SEPEM system database with processed data of solar heavy ions. In the meantime it is worth considering that for a similar component and shielding of $3.7 \mathrm{~mm}$ the PSYCHIC model (Xapsos et al 2007) (as implemented on the SPENVIS system) produces a protoninduced upset rate of 2.85 upsets per bit for a 7-year mission at a $90 \%$ confidence level. With the inclusion of the $>200 \mathrm{MeV}$ 


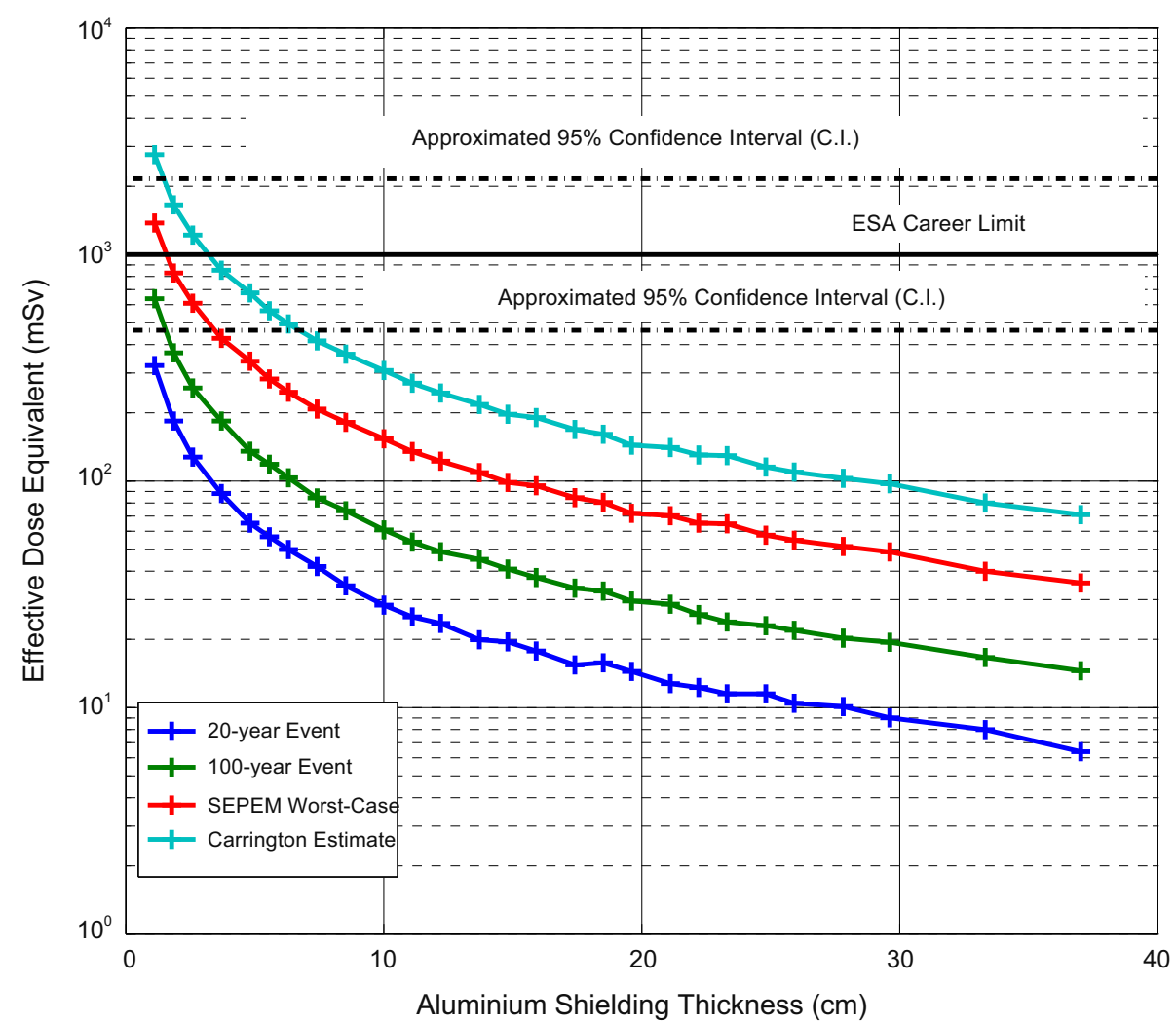

Fig. 13. Effective Dose Equivalent (milli-sieverts) in an astronaut as a result of extreme SPEs compared to the recommended ESA career limit for the four SPEs shown in Figure 9.

protons almost half of these upsets could be single in a single Carrington-class SPE or a quarter in a SEPEM worst-case event. Inclusion of solar heavy ions increases the SEU rate by over four orders of magnitude.

\section{Effects on humans in space}

Effects of radiation on humans in space are divided into stochastic (e.g., cancer-inducing) effects where probability is a function of dose and tissue reactions (e.g., eye cataracts) which will "definitely" occur beyond a threshold dose.

The predicted contributions the four different SPEs would have relative to present career limits put in place to limit stochastic (cancer-causing) effects are shown in Figure 13. The contributions relative to 30-day and annual limits put in place to avoid tissue reactions in the blood-forming organs (BFOs) are shown in Figure 14. The doses were calculated using the 3-D GEANT-4 code for space application: GRAS (Santin et al. 2005).

\subsection{Stochastic effects}

The stochastic effect quantity - the Effective Dose Equivalent, $H_{E}$ - is calculated using the absorbed dose in each organ or tissue, $T$, weighted by a specific mean quality factor, $Q_{T}$, as defined by the ICRP report of 2013 (Dietze et al. 2013):

$$
Q_{T}=\frac{1}{m_{T}} \frac{1}{D_{T}} \int_{m_{T}} \int_{L} Q(L) D_{L} \mathrm{~d} L \mathrm{~d} m,
$$

where $D_{T}$ is the total absorbed dose in the tissue or organ, $T, D(L)$ is the distribution of dose as a function of unrestricted
Linear Energy Transfer (LET), $L, m_{T}$ is the tissue total mass and $Q(L)$ is the quality factor as a function of LET allowing for the inclusion of the relative biological effectiveness of high-LET compared to low-LET radiation. This mean quality factor for a given organ or tissue is then combined with the mean absorbed dose in each tissue or organ, $D_{T}$, and the tissue weighting factor to give the effective dose equivalent:

$$
H_{E}=\sum_{T} w_{T} Q_{T} D_{T}=\sum_{T} \frac{w_{T}}{m_{T}} \int_{m_{T}} \int_{L=0}^{\mathrm{inf}} Q(L) D_{L} \mathrm{~d} L \mathrm{~d} m,
$$

where the tissue weighting factor, $w_{T}$, represents the contribution of one organ or tissue to the overall radiation detriment from stochastic effects. The units of Effective Dose Equivalent are milli-sieverts (mSv).

The foremost concern for stochastic effects is the formation of cancer. As this is a random process and likelihood varies considerably depending on the individual, the limits are calculated based upon a $95 \%$ confidence that there is no more than a $3 \%$ chance of death in the person's lifetime resulting from the radiation encountered while in space - otherwise known as risk of exposure-induced death (REID).

Figure 13 shows the Effective Dose Equivalent values in $\mathrm{mSv}$ resulting from each of the four modelled SPE proton energy spectra as a function of spacecraft shielding. The ESA career limit is presently 1 sievert (Sv) of dose (Straube et al. 2010) as shown in Figure 13. However, there is a large uncertainty in the REID which is used to set this limit, and so a $95 \%$ confidence interval (CI) is also plotted to represent the interval in which one can be $95 \%$ certain that the $3 \%$ REID lies. This uncertainty does not come from the environment or shielding propagation tools, but from the biological effectiveness of the 
P. Jiggens et al.: The magnitude and effects of extreme solor particle events

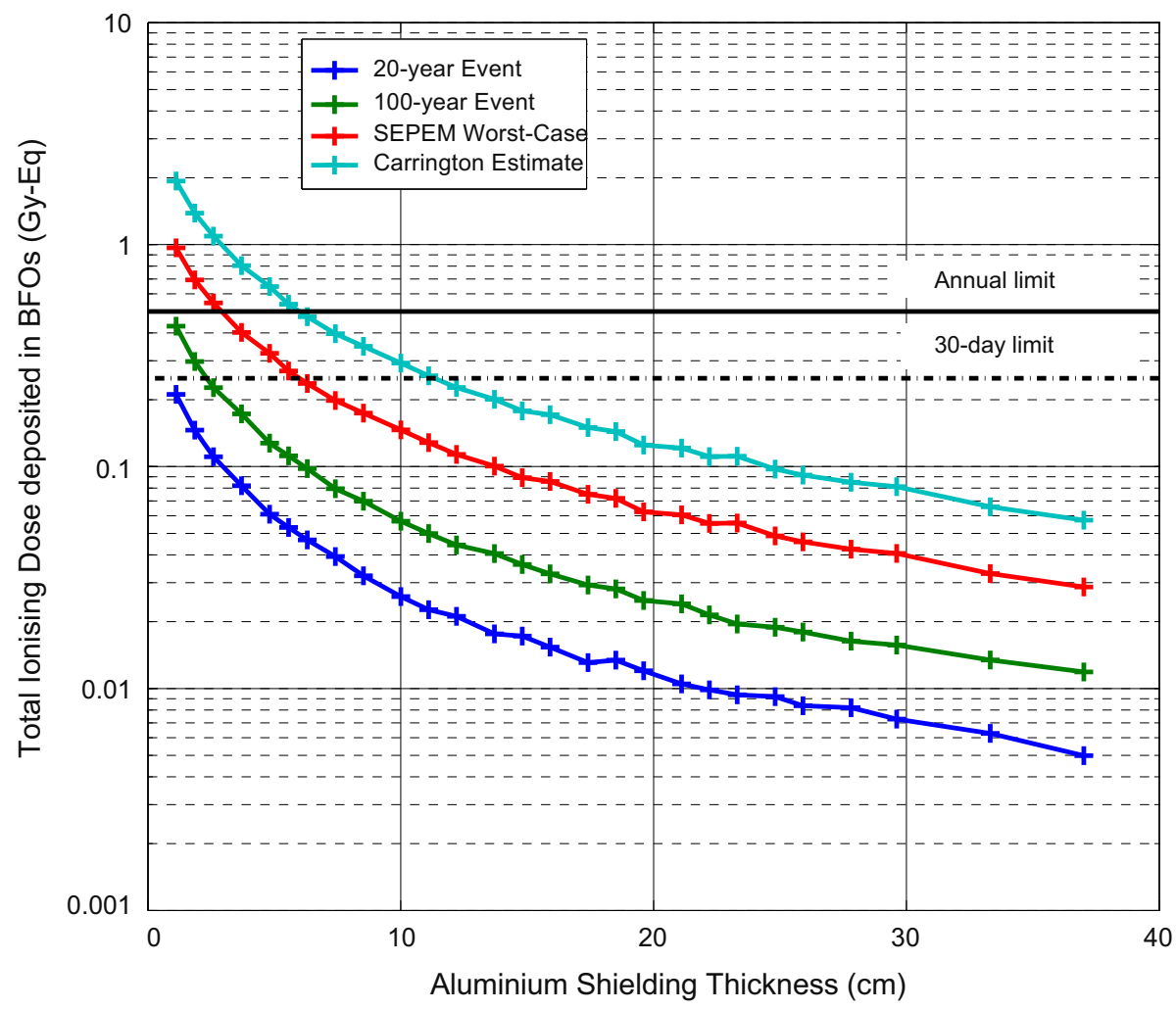

Fig. 14. Total dose deposited (grey-equivalent) in the BFOs as a result of extreme SPEs compared to recommended limits for the four SPEs shown in Figure 9.

radiation, encapsulated in the simple quality factor $Q(L)$ and tissue weights $w_{t}$. The value of 2.16 used to approximate, to first order, the width of the interval is taken from the combined "fold uncertainty" given in Table $6.5 \mathrm{~b}$ in the 2012 NASA report on "Space Radiation Cancer Risk Projections and Uncertainties" (Cucinotta et al. 2013) referring to the uncertainty in radiobiological effects on a 40 -year-old female astronaut during an SPE similar to that of August 1972 behind shielding of $10 \mathrm{~g} \mathrm{~cm}^{-2}$. For a female astronaut of 45 years the NCRP recommended limit is $0.9 \mathrm{~Sv}$ and $0.6 \mathrm{~Sv}$ for 35 years (see Cucinotta et al. 2013, Table 3.1) which is different from the 1 Sv ESA limit. Additionally, the NASA model calculates dose differently than has been done here, further limiting the relevance of NASA-derived uncertainty estimates to the dose results presented above. However, in the absence of a better uncertainty determination, and considering the similarity of both methods and dose values and limits, as well as the highly relevant (arguably dominant) character of REID uncertainties, the inclusion of an approximate CI for REID based on the same factor as the NASA report was deemed reasonable. It is illustrated in Figure 13 as a zone extending above and below the career limit by the same factor, forming a symmetrical, approximate interval.

It appears that even the largest SPE can be relatively easily shielded against although a "SEPEM Worst-Case" or "Carrington" event would contribute a significant dose behind an average $5 \mathrm{~cm} \mathrm{Al}$ shielding especially considering the uncertainty in the REID, and hence approximate CI around the $1 \mathrm{~Sv}$ career dose limit. If experienced on EVA any of these events could result in a significant REID given the uncertainties. Therefore the optimisation of shielding configuration and the need for an adequate warning system to reduce the possibility of astronauts receiving in excess of the recommended dose limit are both very important. If these are carefully considered in the mission planning then the main concern for stochastic effects will come from the low, slowly fluctuating mean flux of high energy GCR particles which are very difficult to shield against. The risk posed by GCRs can be reduced by launching manned missions to fly during solar maximum when these fluxes are reduced due to the increased attenuation in the heliosphere (see Chavy-Macdonald et al. 2013).

\subsection{Tissue reactions}

The TID quantities for tissue reactions have been calculated by weighting the dose deposited in each organ or tissue with the appropriate Relative Biological Effectiveness (RBE) coefficient:

$$
G_{T}=\sum_{R} R B E_{R} D_{T, R}
$$

where the dose deposited in the tissue or organ, $T$, by radiation of type $R$ is given by $D_{T, R}$, The Relative Biological Effectiveness radiation of type $R$ is given by $R B E_{R}$ and $G_{T}$ represents the total dose in organ or tissue of type $T$ given in units of grey-equivalent. The RBE values applied for protons and heavy ions including helium are 1.5 and 2.5 , respectively while values applied for neutrons are $3.5(5-50 \mathrm{MeV})$ and $6.0(1-5 \mathrm{MeV})$. These values are taken from the most recent ICRP report (Dietze et al. 2013).

In Figure 14 the doses deposited in the human bloodforming organs (BFOs) in units of grey-equivalent (Gy-Eq) for the four spectra are given along with the 30-day and 1-year limits (of 0.25 and 0.5 respectively) adopted by ESA. 
It is reported by Wu et al. (2009) that for an extended EVA the recommended 30-day exposure limit would be easily exceeded by an event such as that of August 1972 and that with conventional amounts of spacecraft shielding the early effects of acute radiation sickness (ARS) might not be avoided. The authors state that possible effects that might occur impacting mission success include nausea, vomiting, anorexia, fatigue, skin erythema and epilation and depletion of blood-forming organs. In an extreme case death of an astronaut may be a possibility. It is not thought that an SPE the size of August 1972 would cause death to astronauts behind spacecraft shielding but on EVA the possibility is not excluded and less severe (although still possibly mission-endangering) effects are thought probable. If a Carrington-sized event were an order of magnitude more intense than the August 1972 event then this would have been significantly more damaging in the absence of a storm shelter.

Figure 14 shows that for shielding of $\sim 1 \mathrm{~cm} \mathrm{Al}$ equivalent the ESA annual limit would be exceeded by the SEPEM WorstCase event and that the ESA 30-day limit would be exceeded by the 100 -year SPE and almost by the 20 -year event. That the amount of shielding provided by the weakest region of an EVA suit is 15 times lower than this $\mathrm{Al}$ equivalent (see Wilson et al. 2006) highlights the importance of effective SPE warning for astronauts on EVA to retreat in the spacecraft. Having said this, previous analysis (see Chavy-Macdonald et al., 2013) has shown that inside the Columbus module on-board the International Space Station (ISS) the average shielding is $\sim 30 \mathrm{~g} \mathrm{~cm}^{-2}$ (or $>11 \mathrm{~cm} \mathrm{Al}$ equivalent) which would be sufficient to stay below the ESA 30-day limit for all but the harshest SPE. However, the majority of this shielding is provided by equipment stored on racks on-board ISS and this number may be closer to $\sim 20 \mathrm{~g} \mathrm{~cm}^{-2}$ (or $>7.4 \mathrm{~cm} \mathrm{Al} \mathrm{equivalent)} \mathrm{for} \mathrm{the} \mathrm{space} \mathrm{station}$ in general (Durante \& Cucinotta 2011). Once again, this demonstrates that careful distribution of all spacecraft mass is important for future interplanetary manned mission planning.

A missing detail is that these limits are based on the assumption that the dose is evenly distributed over the entirety of a severe SPE which may last up to 30 days. The flux is not delivered evenly over this period and therefore the dose in the first few days is likely to be disproportionately higher in comparison to the limits if they were scaled down to a shorter time period. Detail on the flux profiles of a selection of large SPEs is given by Neal et al. (2008), this shows that (NASA) limits for deterministic effects are often exceeded within 1-4 days of the SPE onset. Neal et al. also show that the 30-day NASA limit for BFOs (equivalent to the ESA limit) was exceeded by calculated doses in two cases (14 July 2000 and 8 November 2000) in solar cycle 23 even for Al shielding of $3 \mathrm{~g} \mathrm{~cm}^{-2}$ (or $\sim 1.1 \mathrm{~cm}$ ). It should be noted that the GOES fluxes for this work were calibrated with IMP-8/ GME science quality data and this resulted in reduced flux especially at the higher energies. Given this, the results of Neal et al. seem commensurate with the conclusion that a 1-in20 -year event would come very close to exceeding the 30 -day dose limit for a shielding of $1 \mathrm{~cm} \mathrm{Al}$. Note that in the work by Neal et al. (2008) the dose limits are scaled for the RBE values in the plots, whereas in this work the dose quantity is scaled using the RBE values and the dose limits are unchanged.

\section{Discussion}

In this work, four levels of extreme SPEs have been modelled using the SEPEM system, these have been compared to historical events for verification and the effects of these SPEs on components and humans have been estimated.

One major source of error is in the measurements of SPE fluxes. Instruments have caveats which can cause them to mis-report fluxes and while many of these are known about, the corrections made will themselves have an uncertainty. For older data such as those from August 1972 these errors are larger due to inferior instrumentation and less easy to corroborate due to the sparsity of space measurements at the time. The nearEarth interplanetary particle radiation environment (especially protons) has been well covered by measurements since the mid-1970s for the energy ranges which are critical for spacecraft components given nominal shielding thicknesses.

The characterisation of spectra and time evolution of a Carrington-type event such as attempted by McCracken et al. (2001), Smart et al. (2006) and Townsend et al. (2003) would greatly assist the definition of a worst-case event but it appears that thus far this result cannot be achieved reliably. The SEPEM worst-case event (Jiggens et al. 2012) might be an appropriate estimation of a true worst-case but this assumes that the sample of the past 40 years of data are representative of the total population including reasonable sampling of the upper end of the SPE fluence distribution. For these reasons a worst-case $\times 2$ event has been included as an estimate of a Carrington-sized event. It is of interest to note that recently Cliver \& Dietrich (2013) produced an estimate of the Carrington event fluence of $1.1 \times 10^{10} \mathrm{~cm}^{-2}$ for protons of energy $>30 \mathrm{MeV}$ which is identical to the SEPEM Extreme SPE spectra given in Figure 9. However, the uncertainty in that calculation gives the values anywhere between $10^{9} \mathrm{~cm}^{2}$ and $10^{11} \mathrm{~cm}^{2}$ which adequately highlights the problems faced in determining an absolute worst-case SPE.

The higher energies are not as well characterised due to sparsity of measurements and increased uncertainty in the measurements that do exist. Furthermore, SPEs with significant SEP fluxes at higher energies are rarer and therefore the issue of the dataset length is more pronounced. Tylka \& Dietrich (2009) have recently produced spectra for 53 of the 66 GLEs (Ground Level Enhancements) recorded since 1956 and work is ongoing to produce a high energy $(200 \mathrm{MeV}-1 \mathrm{GeV})$ statistical model using these events to extend the model spectra. This will provide better models than the power law extrapolation applied herein. However, the limitation of the dataset size across all energies will remain and extrapolations using models are always dependent upon the solar cycles that have been observed being representative of the long-term population across the distribution of SPE total fluences.

It has been demonstrated that reasonable agreement between methods for calculating total ionising dose and nonionising dose can be found by performing the statistics directly on a time series of effects and running effects tools on the statistical model outputs. The latter method is more useful when the details of the shielding and device are not known while the former is more useful when the design is more mature and allows for focussing the model on the time periods for which the effect is important given the component and geometry. Clearly the data available on the SEPEM system need to be extended upwards in energy and to include heavier ions. This extension is particularly important for SEUs and human effects.

Despite the modelling limitations, the results herein (making use of spectral extrapolations where necessary) are still informative of likely effects as a result of extreme SPEs on components and humans in space. While TID effects do not 
seem to be a concern even for a commercial-grade typical electronic device, the effects of these extreme events might result in a significant performance degradation for unshielded devices (i.e., exposed optics) and in terms of SEU rates and displacement damage, especially at low shielding (i.e., solar cells).

Regarding the human effects, it can be noted that while the "SEPEM Worst-Case" and "Carrington" events might be very dangerous, the $95 \%$ and $99 \%$ confidence interval events can be relatively easily shielded. The most important factors are:

1. A warning system for planned EVAs to ensure that astronauts are not exposed to any large SPEs without significant shielding. Even nominal worst-case SPEs (i.e., the $95 \%$ case studied) could result in radiation sickness jeopardising the mission or contribute an unacceptable amount of dose towards stochastic limits. Oh et al. (2012) present a forecasting system for proton radiation intensity based on data from neutron monitors.

2. Sensible distribution of materials useful for shielding. This would reduce the mass needed for a dedicated storm shelter for major SPEs and should mitigate risks coming from thinly shielded areas for smaller SPEs. A minimum of $10 \mathrm{~cm} \mathrm{Al} \mathrm{equivalent} \mathrm{seems} \mathrm{to} \mathrm{be} \mathrm{an} \mathrm{appropriate} \mathrm{shield-}$ ing level for a storm shelter to protect from extreme SPEs. It is well known that the use of low-atomic mass and high-hydrogen content materials is highly beneficial in terms of reduction of the spacecraft mass and dose equivalent exposure (Wilson et al. 1999).

\section{References}

Adams, J.H., Cosmic ray effects on microelectronics, part 4, Technical Report Report 5901, Naval Research Laboratory, 1986.

Badhwar, G.D., and P.M. O'Neill, Galactic cosmic radiation model and its applications, Adv. Space Res., 17 (2), 7-17, 1996.

Carrington, R.C., Description of a singular appearance seen on the Sun on September 1, 1859, Monthly Notices Royal Astronomical Society, 20, 13-15, 1860.

Chavy-Macdonald, M.-A., A. Menicucci, G. Santin, H. Evans, P.T.A. Jiggens, P. Nieminen, and S. Hovland, High-accuracy simulations of the ISS radiation environment and applications to interplanetary manned missions, IEEE Trans. Nucl. Sci., 60 (4), 2427-2434, 2013.

Clauer, R.C., and G. Siscoe, The great historical geomagnetic storm of 1859: a modern look, Adv. Space Res., 38, 117-118, 2006.

Cliver, E.W., and W.F. Dietrich, The 1859 space weather event revisited: limits of extreme activity, Journal of Space Weather and Space Climate, 3 (A31), 121-124, 2013.

Cucinotta, F.A., M.-H.Y. Kim, and L.J. Chappell, NASA/TP-2013217375: Space radiation cancer risk projections and uncertainties 2012. In: Technical report, National Aeronautics and Space Administration, 2013.

Dietze, G., D.T. Bartlett, D.A. Cool, F.A. Cucinotta, X. Jia, I.R. McAulay, M. Pelliccioni, V. Petrov, G. Reitz, and T. Sato, Annals of the ICRP: Assessment of radiation exposure of astronauts in space, The International Commission on Radiological Protection, 42 (4), 1-339, 2013.

Dorman, L.I., L.I. Pustilnik, A. Sternlieb, I.G. Zukerman, A.V. Belov, E.A. Eroshenko, V.G. Yanke, H. Mavromichalaki, C. Sarlanis, G. Souvatzoglou, S. Tatsis, N. Iucci, G. Villoresi, Y. Fedorov, B.A. Shakhov, and M. Murat, Monitoring and forecasting of great solar proton events using the neutron monitor network in real time, IEEE Transactions on Plasma Science, 32 (4), 1478-1488, 2004
Durante, M., and F.A. Cucinotta, Physical basis of radiation protection in space travel, Rev. Mod. Phys., 83 (4), 1245-1281, 2011.

Gopalswamy, N., S. Yashiro, S. Krucker, G. Stenborg, and Russell A. Howard, Intensity variation of large solar energetic particle events associated with coronal mass ejections, J. Geophys. Res., 109 (A12105), 1-18, 2004.

ISO 15390, Space Environment (natural and artificial) - Galactic Cosmic Ray model, 2004

Jiggens, P.T.A., and S.B. Gabriel, Time distributions of solar energetic particle events: Are SEPEs really random? J. Geophys. Res., 114 (A10), A10105, 2009.

Jiggens, P.T.A., S.B. Gabriel, D. Heynderickx, N. Crosby, A. Glover, and A. Hilgers, ESA SEPEM project: peak flux and fluence model, IEEE Trans. Nucl. Sci., 59 (4), 1066-1077, 2012.

Jun, I., M.A. Xapsos, S.R. Messenger, E.A. Burke, R.J. Walkter, and T. Jordan, Nonionizing energy loss (NIEL) for device applications, IEEE Trans. Nucl. Sci., 50 (6), 1924-1928, 2003.

Kahler, S.W., The correlation between solar energetic particle peak intensities and speeds of coronal mass ejections: effects of ambient particle intensities and energy spectra, J. Geophys. Res., 106, 20947-20955, 2001.

Kahler, S.W., Energetic particle acceleration by coronal mass ejections, Adv. Space Res., 32 (12), 2587-2596, 2003.

Kim, M.-H.Y., K.A. George, and F.A. Cucinotta, Evaluation of skin cancer risk for lunar and mars missions, Adv. Space Res., 37, 1798-1803, 2006.

King, J.H., Solar proton fluences for 1977-1983 space missions, $J$. Spacecraft Rockets, 11 (6), 401-408, 1974.

Lario, D., M.B. Kallenrode, R.B. Decker, E.C. Roelof, S.M. Krimigis, A. Angels, and S. Blai, Radial and longitudinal dependences of solar 4-13 Mev and 27-37 Mev proton peak intensities and fluences: helios and imp-8 observations, Astrophys. J., 653, 1531-1544, 2006.

Lei, F., P.R. Truscott, C.S. Dyer, B. Quaghebeur, D. Heynderickx, P. Nieminen, H. Evans, and E. Daly, MULASSIS: a Geant4-based multilayered shielding simulation tool, IEEE Trans. Nucl. Sci., 49 (6), 2788-2793, 2002.

McCracken, K.G., G.A.M. Dreschhoff, E.J. Zeller, D.F. Smart, and M.A. Shea, Solar cosmic ray events for the period 1561-1994: 1 Identification in polar ice 1561-1950, J. Geophys. Res. - Space Phys., 106 (A10), 21585-21598, 2001.

Mewaldt, R.A., C.M.S. Cohen, A.W. Labrador, R.A. Leske, G.M. Mason, M.I. Desai, M.D. Looper, J.E. Mazur, R.S. Selesnick, and D.K. Haggerty, Proton, helium, and electron spectra during the large solar particle events of October-November 2003, J. Geophys. Res., 110, A09S18, 2005.

Neal, J.S., T.F. Nichols, and L.W. Townsend, Importance of predicting the dose temporal profile for large solar energetic particle events, Space Weather, 6 (1), S09004, 2008.

Nymmik, R.A., The lag of galactic cosmic ray modulation: conformity to general regularities and influence on particle energy spectra, Adv. Space Res., 26 (11), 1875-1878, 1996.

Nymmik, R.A., Improved environment radiation models, $A d v$. Space Res., 40, 313-320, 2007.

Nymmik, R.A., M.I. Panasyuk, and A.A. Suslov, Galactic cosmic ray flux simulation and prediction, Adv. Space Res., 17 (2), 19-30, 1996.

Oh, S.Y., J.W. Bieber, J. Clem, P. Evenson, R. Pyle, Y. Yi, and Y.-K. Kim, South pole neutron monitor forecasting of solar proton radiation intensity, Space Weather, 10, S05004, 2012.

O’Neill, P.M., Badhwar-O'Neill galactic cosmic ray model update based on advanced composition explorer (ace) energy spectra from 1997 to present, Adv. Space Res., 37, 1727-1733, 2006.

O'Neill, P.M., Badhwar-O'Neill 2010 galactic cosmic ray flux model - revised, IEEE Trans. Nucl. Sci., 57, 3148-3153, 2010.

Petersen, E.L., The SEU figure of merit and proton upset rate calculations, IEEE Trans. Nucl. Sci., 45 (6), 2550-2562, 1998. 
Rosenqvist, L., and A. Hilgers, Sensitivity of a statistical solar proton fluence model to the size of the event data set, Geophys. Res. Lett., 30 (16), 1-4, 2003.

Santin, G., V. Ivanchenko, E. Evans, P. Nieminen, and E. Daly, GRAS: a general-purpose 3-D modular simulation tool for space environment effects analysis, IEEE Trans. Nucl. Sci., 52 (6), 2294-2299, 2005.

Seltzer, S.M., Updated calculations for routine space-shielding radiation dose estimates: SHIELDOSE-2, NIST Publication, NISTIR 5477, 1994.

Shea, M.A., and D.F. Smart, A summary of solar proton events, Solar Phys., 127, 297-320, 1990.

Siscoe, G., N.U. Crooker, and C.R. Clauer, Dst of the Carrington storm of 1859, Adv. Space Res., 38, 173-179, 2006.

Smart, D.F., M.A. Shea, and K.G. McCracken, The Carrington event: possible solar proton intensitytime profile, Adv. Space Res., 38, 215-225, 2006

Stapor, W.J., J.P. Meyers, J.B. Langworthy, and E.L. Petersen, Two parameter Bendel model calculations for predicting proton induced upset, IEEE Trans. Nucl. Sci., 37 (6), 1966-1973, 1990.

Straube, U., T. Berger, G. Reitz, R. Facius, C. Fuglesang, T. Reiter, V. Damann, and M. Tognini, Operational radiation protection for astronauts and cosmonauts and correlated activities of ESA medical operations, Acta Astronaut., 66 (7-8), 963-973, 2010.

Technical Standard NASA-STD-3001, Nasa space flight human system standard volume 1: Crew health. Technical report, National Aeronautics and Space Administration, 2007.

Townsend, L.W., E.N. Zapp, D.L. StephenJr., and J.L. Hoff, Carrington flare of 1959624 as a prototypical worst-case solar energetic particle event, IEEE Trans. Nucl. Sci., 50 (6), 23072309,2003

Tylka, A.J., and W.F. Dietrich, A new and comprehensive analysis of proton spectra in ground-level enhanced (GLE) solar particle events, 31st International Cosmic Ray Conference, Łódź, 2009.
Tylka, A.J., J.H. Adams, P.R. Boberg, B. Brownstein, W.F. Dietrich, E.O. Flueckiger, E.L. Peterson, M.A. Shea, D.F. Smart, and E.C. Smith, Creme96: A revision of the cosmic ray effects on microelectronics code, IEEE Trans. Nucl. Sci., 44 (6), 2150-2160, 1997.

Wilson, J.W., F.A. Cucinotta, J.L. Shinn, L.C. Simonsen, R.R. Dubey, W.R. Jordan, T.D. Jones, C.K. Chang, and M.Y. Kim, Shielding from solar particle event exposures in deep space, Radiat. Meas., 30 (3), 361-382, 1999.

Wilson, J.W., F.A. Cucinotta, and C.J. Zeitlin, Spacesuit radiation shield design methods, 36th International Conference on Environmental Systems (ICES), Norfolk, Virginia, US, 2006.

Wolff, E.W., M. Bigler, M.A. Curran, J.E. Dibb, M.M. Frey, M.R. Legrand, and J.R. McConnell, The Carrington event not observed in most ice core nitrate records, Geophys. Res. Lett., 39, L08503, 2012.

Wu, H., J.L. Huff, R. Casey, M.-H. Kim, and F.A. Cucinotta, Chapter 5: risk of acute radiation syndromes due to solar particle events. In: J.C., McPhee, and J.B. Charles (Eds.), Human Health and Performance Risks of Space Exploration Missions, US, NASA, Lyndon B. Johnson Space Center, pp. 171-190, 2009.

Xapsos, M.A., G.P. Summers, J.L. Barth, E.G. Stassinopoulos, and E.A. Burke, Probability model for worst case solar proton event fluences, IEEE Trans. Nucl. Sci., 46 (6), 1481-1485, 1999.

Xapsos, M.A., J.L. Barth, E.G. Stassinopoulos, S.R. Messenger, R.J. Walters, G.P. Summers, and E.A. Burke, Characterizing solar proton energy spectra for radiation effects applications, IEEE Trans. Nucl. Sci., 47 (6), 2218-2223, 2000.

Xapsos, M.A., C. Stauffer, T. Jordan, J.L. Barth, and R.A. Mewaldt, Model for cumulative solar heavy ion energy and linear energy transfer spectra, IEEE Trans. Nucl. Sci., 54 (6), 1985-1989, 2007.

Cite this article as: Jiggens P, Chavy-Macdonald MA, Santin G, Menicucci A, Evans H, Hilgers A: The magnitude and effects of extreme solar particle events. J. Space Weather Space Clim., 2014, 4, A20. 